RECEIVED

JAN 301996

OSTI
WHC-SP-1133.

UC-900

\title{
Materials Management FY 1995 Site Support Program Plan WBS 6.10.7
}

E. C. Dahlin

Date Published

September 1994

Prepared for the U.S. Department of Energy

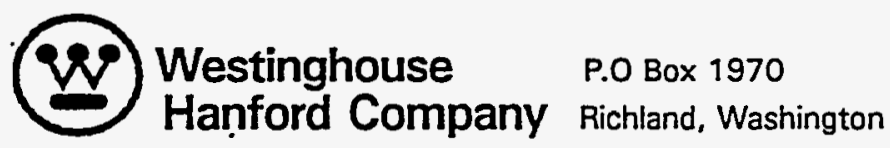

Hanford Operations and Engineering Contractor for the

U.S. Department of Energy under Contract DE-ACO6-87RL10930

Approved for Public Release 
LEGAL DISCLAIMER

This report was prepared as an account of work sponsored by an agency of the United States Government. Neither the United States Government nor any agency thereof, nor any of their employees, nor any of their contractors, subcontractors or their employees, makes any warranty. express or implied, or assumes any legal liability or responsibility for the accuracy, completeness, or any third party's use or the results of such use of any information, apparafus, product, or process disclosed. or represents that its use would not iniringe privately owned rights. Relerence herein to any specific commercial product, process, or service by trade name. trademark, manufacturer, or otherwise, does not necessarily constitute or imply its endorsement, recommendation, or favoring by the United States Government or any agency thereof or its contractors or subcontractors. The views and opinions of authors expressed herein do not necessarily state or reflect those of the United States Government or any agency thereof.

This report has been reproduced from the best available copy. Available in paper copy and microfiche.

Available to the U.S. Department of Energy and its contractors from

Office of Scientific and Technical Information

P.O. Box 62

Oak Ridge, TN 37831

(615) 576.8401

Available to the public from the U.S. Department of Commerce National Technical Information Service

5285 Port Royal Road

Springfield, VA 22161

(703) $487-4650$

Printed in the United States of America

DISCLM-1.CHP (1-91) 
RELEASE AUTHORIZATION

Document Number: WHC-SP-1133, Rev. 0

Document Title: Materials Management FY 1995 Site Support Program P1 an WBS 6.10.7

Release Date: · $09 / 22 / 95$

This document was reviewed following the procedures described in WHC-CM-3-4 and is:

APPROVED FOR PUBLIC RELEASE

WHC Information Release Administration Specialist:

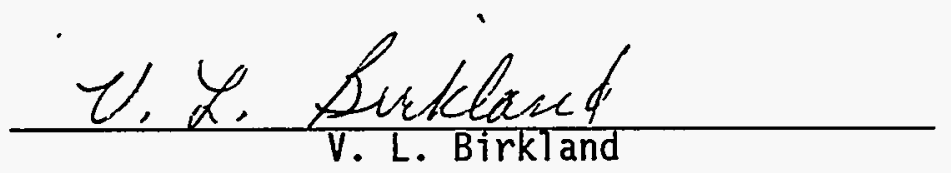

$$
9 / 22 / 95
$$

A-6001-400.2 (09/94) WEF256 


\title{
Site Support Program Plan Approval Sheet
}

\author{
6.10.7 MATERIALS MANAGEMENT
}

Assistant Manager-Contracting Officer's Representative

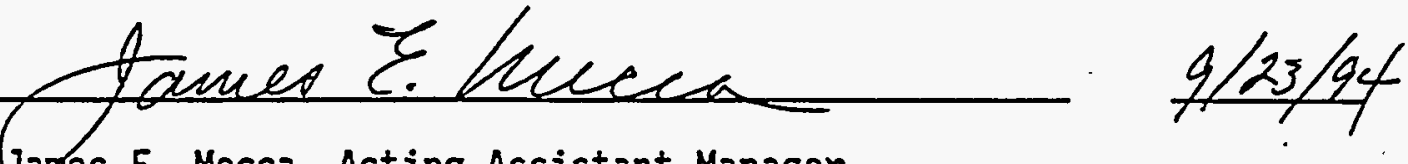

Janes E. Mecca, Acting Assistant Manager

Facility Transition and Infrastructure
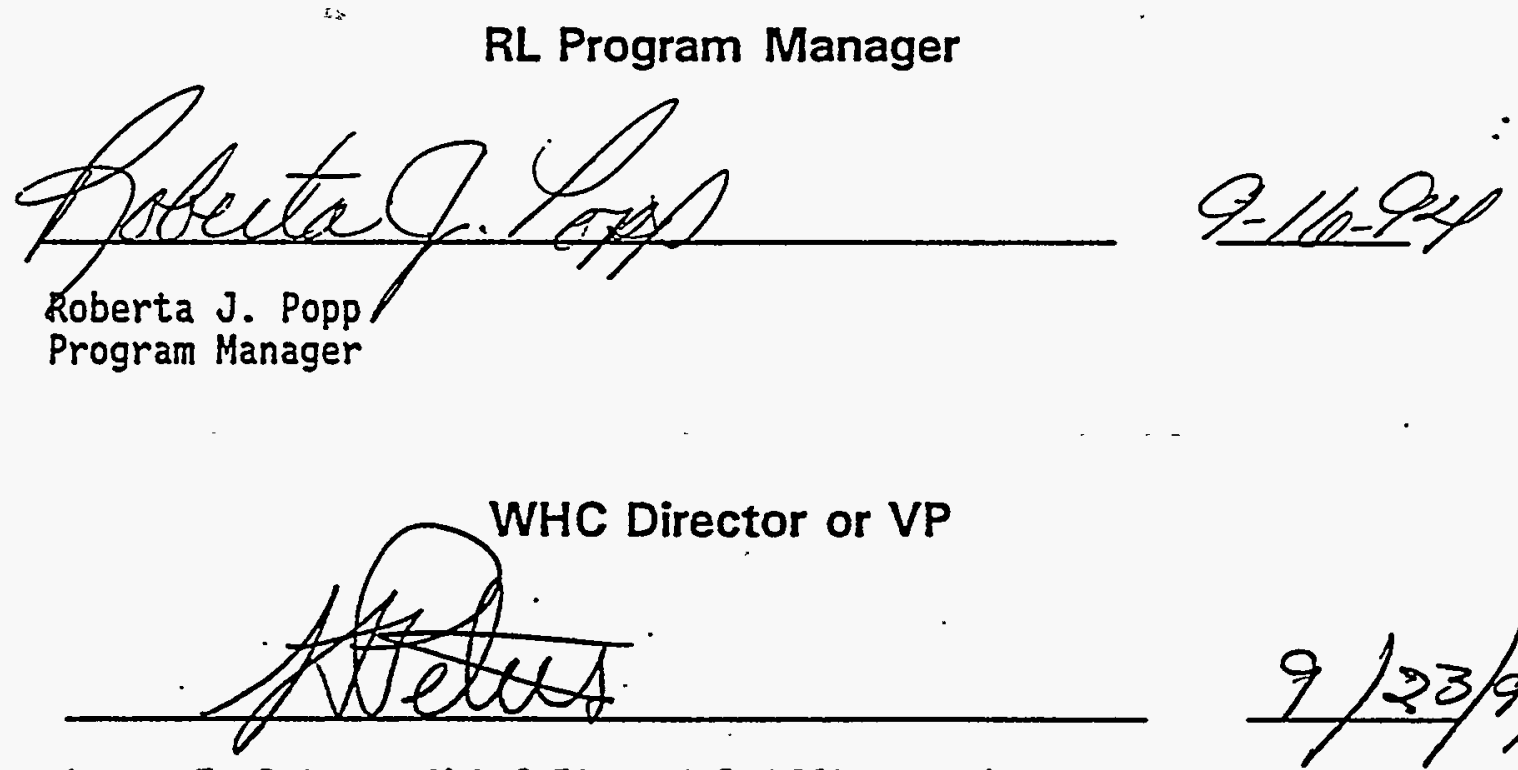

Larry F. Peters, Chief Financial Officer and

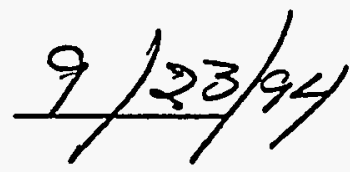

Manager of Administration
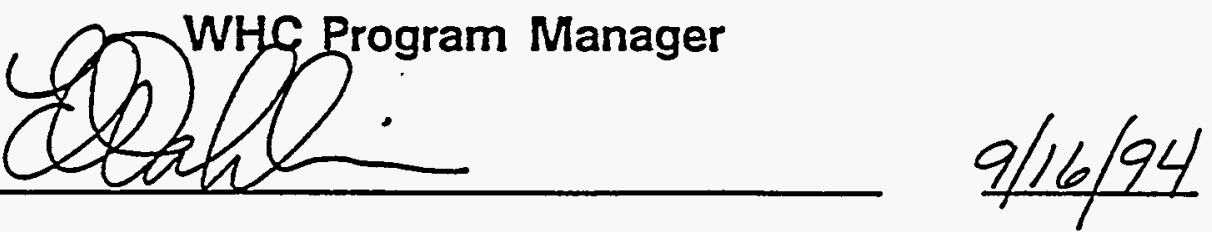

Eric C. Dahlin, Manager, Materials Management 


\begin{tabular}{|c|c|c|c|c|c|}
\hline $\begin{array}{l}\text { Item } \\
\text { Number }\end{array}$ & Title & Description of Content & $\begin{array}{l}\text { Frequency } \\
\text { of } \\
\text { Issuance } \\
\end{array}$ & Due Date & $\begin{array}{r}\text { Approval } \\
\text { Requirements } \\
\end{array}$ \\
\hline 1 & $\begin{array}{l}\text { Site Management Systems } \\
\text { (SMS) Report }\end{array}$ & $\begin{array}{l}\text { Report of progress on } 1995 \\
\text { Fiscal Year Work Plan. }\end{array}$ & Monthly & $\begin{array}{l}\text { 3rd Friday } \\
\text { of Fiscal } \\
\text { Month }\end{array}$ & $\begin{array}{l}\text { Manager } \\
\text { Materials } \\
\text { Management }\end{array}$ \\
\hline 2 & $\begin{array}{l}\text { Loss/Damage/Destruc- } \\
\text { tion Report }\end{array}$ & $\begin{array}{l}\text { Report of items that are } \\
\text { lost, damaged or destroyed } \\
\text { including the circumstances } \\
\text { and administrative actions } \\
\text { to prevent recurrence. }\end{array}$ & $\begin{array}{l}\text { As } \\
\text { occurring }\end{array}$ & $\begin{array}{l}\text { As } \\
\text { occurring }\end{array}$ & $\begin{array}{l}\text { Manager, } \\
\text { Materiais } \\
\text { Management }\end{array}$ \\
\hline 3 & $\begin{array}{l}\text { DOE Precious Metals } \\
\text { Program, Require- } \\
\text { ments for Precious } \\
\text { Metals }\end{array}$ & $\begin{array}{l}\text { Three year forecast of } \\
\text { precious metals require- } \\
\text { ments. }\end{array}$ & Annual & August & $\begin{array}{l}\text { Manager, } \\
\text { Materials } \\
\text { Managemenl. }\end{array}$ \\
\hline 4 & Precious Metals Review & $\begin{array}{l}\text { Review custodian management } \\
\text { precious metals for records } \\
\text { completeness and accuracy, } \\
\text { physical protection, com- } \\
\text { pliance with requirements, } \\
\text { and follow-up on prior } \\
\text { action recommendations. }\end{array}$ & of Annual & September & $\begin{array}{l}\text { Manager, } \\
\text { Materiais } \\
\text { Management }\end{array}$ \\
\hline 5 & $\begin{array}{l}\text { Report on Activities } \\
\text { Generating Precious } \\
\text { Metals }\end{array}$ & $\begin{array}{l}\text { Report of recovery of } \\
\text { precious metals from such } \\
\text { sources as items excessed } \\
\text { and returned to the Precious } \\
\text { Metals Pool and silver re- } \\
\text { covered from photographic } \\
\text { film and hypo solution. }\end{array}$ & Annual & October & $\begin{array}{l}\text { Manager, } \\
\text { Materials } \\
\text { Management }\end{array}$ \\
\hline
\end{tabular}




\begin{tabular}{|c|c|c|c|c|c|}
\hline $\begin{array}{l}\text { Item } \\
\text { Number }\end{array}$ & Title & Description of Content & $\begin{array}{l}\text { frequency } \\
\text { of I ssuance }\end{array}$ & Due Date & $\begin{array}{l}\text { Approval } \\
\text { requirements }\end{array}$ \\
\hline 6 & $\begin{array}{l}\text { Report of Excess } \\
\text { Personal Property } \\
\text { Furnished to Non- } \\
\text { Federal Recipients }\end{array}$ & $\begin{array}{l}\text { Report of excess govern- } \\
\text { ment personal property } \\
\text { acquired by. Wlic from } \\
\text { Federal Agencies or DOE } \\
\text { direct operations. }\end{array}$ & Annual & October & $\begin{array}{l}\text { Manager, } \\
\text { Materiais } \\
\text { Management }\end{array}$ \\
\hline 7 & $\begin{array}{l}\text { Management of Equip- } \\
\text { ment Held for Future } \\
\text { Projects (EHFFP) }\end{array}$ & $\begin{array}{l}\text { Report of Equipment lield } \\
\text { for Future Projects } \\
\text { which has been retained } \\
\text { for three years or } \\
\text { Tonger. }\end{array}$ & Annual & November & $\begin{array}{l}\text { Manager, } \\
\text { Materiais } \\
\text { Management }\end{array}$ \\
\hline 8 & $\begin{array}{l}\text { Site Support Program } \\
\text { Plan }\end{array}$ & $\begin{array}{l}\text { Fiscal Year Work Plan } \\
\text { for the coming year. }\end{array}$ & Annual & September & $\begin{array}{l}\text { Manager, } \\
\text { Materiais } \\
\text { Management }\end{array}$ \\
\hline 9 & Walkthrough Plan & $\begin{array}{l}\text { Plan for Property Man- } \\
\text { agement Walkthroughs }\end{array}$ & Annual & $\begin{array}{l}\text { Last work } \\
\text { day of } \\
\text { September }\end{array}$ & $\begin{array}{l}\text { Manager, } \\
\text { Materiais } \\
\text { Management }\end{array}$ \\
\hline 10 & Walkthrough Reports & $\begin{array}{l}\text { Report Property Manage- } \\
\text { ment Walkthrough obser- } \\
\text { vations, findings and } \\
\text { responses }\end{array}$ & $\begin{array}{l}30 \text { days } \\
\text { after } \\
\text { walkthrough }\end{array}$ & $\begin{array}{l}30 \text { days } \\
\text { after } \\
\text { walkthrough }\end{array}$ & $\begin{array}{l}\text { Manager, } \\
\text { Materials } \\
\text { Management }\end{array}$ \\
\hline 11 & Inventory Schedule & $\begin{array}{l}\text { Plan for physical in- } \\
\text { ventory of government } \\
\text { property }\end{array}$ & Annual & $\begin{array}{l}\text { Last work } \\
\text { day of } \\
\text { September }\end{array}$ & $\begin{array}{l}\text { Manager, } \\
\text { Materials } \\
\text { Management }\end{array}$ \\
\hline
\end{tabular}

Materials Management Page 22 


\begin{tabular}{|c|c|c|c|c|c|}
\hline $\begin{array}{l}\text { Item } \\
\text { Number }\end{array}$ & Title & \begin{tabular}{l|l} 
Description of & Fr \\
Content & of
\end{tabular} & $\begin{array}{l}\text { Frequency } \\
\text { of Is suance }\end{array}$ & Due Date & $\begin{array}{l}\text { Approval } \\
\text { Requirements }\end{array}$ \\
\hline 12 & Inventory Report & $\begin{array}{l}\text { Report closed inventor- } \\
\text { ies for the month and } \\
\text { Fiscal Year to date in- } \\
\text { cluding numbers and } \\
\text { percentage of items and } \\
\text { value inventoried, } \\
\text { written off and added. }\end{array}$ & Monthly & $\begin{array}{l}\text { Last working } \\
\text { day of the } \\
\text { month re- } \\
\text { ported }\end{array}$ & $\begin{array}{l}\text { Manager, } \\
\text { Materiais } \\
\text { Management }\end{array}$ \\
\hline 13 & $\begin{array}{l}\text { Sensitive Property } \\
\text { Consumption Review } \\
\text { Report }\end{array}$ & $\begin{array}{l}\text { Report the results of } \\
\text { sensitive stores \& } \\
\text { direct purchased item } \\
\text { consumption. }\end{array}$ & $\begin{array}{l}\text { Semi- } \\
\text { Annual }\end{array}$ & $\begin{array}{l}\text { January } \\
\text { \& July }\end{array}$ & $\begin{array}{l}\text { Manager, } \\
\text { Materials } \\
\text { Management }\end{array}$ \\
\hline 14 & $\begin{array}{l}\text { Report of Numbers } \\
\text { and Value of } \\
\text { Personal Property } \\
\text { Items }\end{array}$ & $\begin{array}{l}\text { Report numbers and value } \\
\text { of Fixed Capital, Movable } \\
\text { Capital, Sensitive, Non- } \\
\text { Capital/Non-Sensitive and } \\
\text { Equipment Held for Future } \\
\text { Projects items. }\end{array}$ & $\begin{array}{l}\text { Semi- } \\
\text { Annual }\end{array}$ & $\begin{array}{l}\text { March \& } \\
\text { September }\end{array}$ & None \\
\hline 15 & $\begin{array}{l}\text { Property Manage- } \\
\text { ment Articles }\end{array}$ & $\begin{array}{l}\text { Publish Articles, notices } \\
\text { bulletins on appropriate } \\
\text { property management } \\
\text { subjects }\end{array}$ & Quarterly & $\begin{array}{l}\text { December, } \\
\text { March, } \\
\text { June, } \\
\text { September }\end{array}$ & $\begin{array}{l}\text { Manager, } \\
\text { Materiais } \\
\text { Management }\end{array}$ \\
\hline 16 & Recycling Articles & $\begin{array}{l}\text { Articles promoting recyc- } \\
\text { ling and conservation } \\
\text { efforts. }\end{array}$ & $\begin{array}{l}\text { Bi- } \\
\text { Monthly }\end{array}$ & $\begin{array}{l}\text { Bi- } \\
\text { Monthly }\end{array}$ & $\begin{array}{l}\text { Manager, } \\
\text { Excess, } \\
\text { Surplus } \\
\text { Sales \& } \\
\text { Shipping }\end{array}$ \\
\hline
\end{tabular}

Materials Management Page 23 
$1 . G$

\begin{tabular}{|l|l|l|l|l|l||}
\hline $\begin{array}{l}\text { Item } \\
\text { Number }\end{array}$ & Title & $\begin{array}{l}\text { Description of } \\
\text { Content }\end{array}$ & $\begin{array}{l}\text { Frequency of } \\
\text { Issuance }\end{array}$ & $\begin{array}{l}\text { Due } \\
\text { Date }\end{array}$ & $\begin{array}{l}\text { Approval } \\
\text { Requirements }\end{array}$ \\
\hline
\end{tabular}

17

Community Outreach

Stores Inventory Performance Indi-

Precious Metal

call letter

Annual Report of

\author{
Participation in School \\ Ambassador Program, \\ Earth Day, and other \\ activities that support \\ and publicize llanford \\ recycle efforts.
} Fixed Price Sales

tion on General

Supplies Inventory

value, turnover,

service level, month/

year supply on hand,

Tine items and excess

activity \&; (b) infor-

mation on excess re-

distribution and sale

activity.

tractors to forecast

anticipated volume of

excess precious metals. activity provided to the
Semi-Annual

March \&

September

Excess

Surplits

Sales \&

Shipping

Includes (a) informa- Quarterly

Request all Hanford Con- Annual

Yearly fixed price sales Annual General Services Adninistration

in Auburn. 15th work- Manager, day of the Procurement \&

month Materials

following Management (PMM)

the last

day of the

quarter.

January Manager, PMM

$\begin{array}{ll}\text { October } & \text { Manager, } \\ & \text { Excess, } \\ \text { Surplus } \\ \text { Sales \& } \\ \text { Shipping }\end{array}$

Materials Management Page 24 
$1 . G$

\begin{tabular}{|l|l|l|l|l|l|}
\hline $\begin{array}{l}\text { Item } \\
\text { Number }\end{array}$ & Title & Description of .Content & $\begin{array}{l}\text { Frequency of } \\
\text { Issuance }\end{array}$ & Due Date & $\begin{array}{l}\text { Approval } \\
\text { Requirements }\end{array}$ \\
\hline
\end{tabular}

21 General Supplies Inventory Control

Automate cycle counting

within the Warehouse

$N / A$

Inventory Management System

$01 / 30 / 95$

General Supplies

Inventory

Manager.

22

Utilization \& Disposal

of Excess \& Surplus

Report \#CR-85-2, provides

Annual

Last work-
ing day of
October

annual suminary of the

utilization \& disposal of

excess \& surplus personal

property.

Manager,

Excess,

Surplus

Sales \&

Shipping

23

Allowable Loss Rates for Financially Report of physical inventory Annual
losses of Precious Metals and

November

Manager,

Materials

Controlled Inventories

Financially Controlled

Management

Inventories.

24 Materials Management Training Summary

Quarterly summary of training Quarterly provided to and by Materials Management Personnel to enhance safety awareness, skill levels and customer awareness.

15 th work Manager, day of the Materials month Management

following the

last day of

the quarter.

Mathematics and Science Education Gift Program

Annual report of property Annual

Last work day of October

Manager, gifted to primary and secondary schools in support of the Mathematics and Science Education Excess, Gift program.

Surplus

Sales \&

Shipping 


\begin{tabular}{|c|c|c|c|c|c|}
\hline \multicolumn{2}{|l|}{$\begin{array}{l}2 . \text { A. } 1 \\
\text { Work } \\
\text { Breakdown } \\
\text { Structure and } \\
\text { Responsibility } \\
\text { Assignment } \\
\text { Matrix }\end{array}$} & \multicolumn{2}{|c|}{$\begin{array}{c}\text { West inghouse Hanford Company } \\
\text { Materials Management } \\
\text { SMS/WBS No. } 6.10 .7\end{array}$} & \multicolumn{2}{|c|}{$\begin{array}{c}\text { FY } 1995 \\
\text { Site Support Program Plan } \\
\text { Date Prepared:06/28/94 }\end{array}$} \\
\hline Program Element & $\begin{array}{c}\text { Activity/Program } \\
\text { Element }\end{array}$ & Cost Account & Title & $\begin{array}{c}\text { Responsible } \\
\text { Manager }\end{array}$ & $\begin{array}{c}\text { Responsible } \\
\text { Organization } \\
\end{array}$ \\
\hline $\begin{array}{l}\text { Materials } \\
\text { Management }\end{array}$ & $\begin{array}{l}\text { Materials } \\
\text { Management } \\
6.10 .7 \\
\end{array}$ & & $\begin{array}{l}\text { Materials } \\
\text { Management }\end{array}$ & E. C. Dahl in & $\begin{array}{l}\text { Materials } \\
\text { Management }\end{array}$ \\
\hline \multirow[t]{3}{*}{$\cdot$} & $\begin{array}{l}\text { Property } \\
\text { Management } \\
6.10 .7 .1 \\
\end{array}$ & & $\begin{array}{l}\text { Property } \\
\text { Management }\end{array}$ & $\begin{array}{l}\text { T.J. } \\
\text { Christensen } \\
\text { J.L. Utz }\end{array}$ & $\begin{array}{l}\text { Property Records } \\
\text { \& Property } \\
\text { Utilization }\end{array}$ \\
\hline & $\begin{array}{l}\text { Warehousing } \\
6.10 .7 .2\end{array}$ & & Warehousing & $\begin{array}{l}\text { J.B. Paris } \\
\text { E.E. } \\
\text { Fairchild } \\
\text { H.C. } \\
\text { Humphrey } \\
\text { G.W. Sandall }\end{array}$ & $\begin{array}{l}\text { Central } \\
\text { Warehouse } \\
\text { Operations } \\
100 \text { Area SWS } \\
200 \text { Area SWS } \\
3 / 400 \text { Area SWS }\end{array}$ \\
\hline & $\begin{array}{l}\text { Inventory } \\
\text { Management } \\
6.10 .7 .3 \\
\end{array}$ & & $\begin{array}{l}\text { Inventory } \\
\text { Management }\end{array}$ & $\begin{array}{l}\text { J. G. } \\
\text { Caudill } \\
\text { R. E. Boykin }\end{array}$ & $\begin{array}{l}\text { SCMT } \\
\text { Inventory Mgmt. }\end{array}$ \\
\hline \multirow[t]{2}{*}{. } & $\begin{array}{l}\text { Investment } \\
\text { Recovery } \\
6.10 .7 .4 \\
\end{array}$ & & $\begin{array}{l}\text { Investment } \\
\text { Recovery }\end{array}$ & $\begin{array}{l}\text { G. B. } \\
\text { Carlson }\end{array}$ & $\begin{array}{l}\text { Excess, Surplus } \\
\text { Sales and } \\
\text { Shipping }\end{array}$ \\
\hline & $\begin{array}{l}\text { Property } \\
\text { Systems: } \\
6.10 .7 .5 \\
\end{array}$ & & Property Systems & $\begin{array}{l}\text { J. N. } \\
\text { Delahunt }\end{array}$ & Material Systems \\
\hline
\end{tabular}




\begin{tabular}{|c|c|}
\hline 2.A.2 Description of Activities & $\begin{array}{l}\text { Westinghouse Hanford Company } \\
\text { Materials Management } \\
\text { SMS/WBS No. 6.10.7 }\end{array}$ \\
\hline ACTIVITY & DESCRIPTION \\
\hline Materials Management & $\begin{array}{l}\text { Provide the Hanford Site with a safe, environmentally sound, and } \\
\text { cost effective program for the receipt, storage, issuance, } \\
\text { management and disposition of the government's physical assets. }\end{array}$ \\
\hline Property Management & $\begin{array}{l}\text { Provide the systems, services, and management controls required by } \\
\text { Federal and DOE regulations to maintain acceptable levels of } \\
\text { property accountability and proper utilization of government owned } \\
\text { property. }\end{array}$ \\
\hline Warehousing & $\begin{array}{l}\text { Provide facilities, trained personnel, services, and management } \\
\text { controls for the shipping, receiving, storage, issuance, and } \\
\text { distribution of materials, parts, components and equipment required } \\
\text { to support the ongoing operation of the Hanford site. }\end{array}$ \\
\hline Inventory Management & $\begin{array}{l}\text { Provide inventory management services to maintain appropriate } \\
\text { levels of general supplies, spare parts, and essential materials to } \\
\text { ensure availability of items required to support site operations is } \\
\text { timely and provided at the lowest possible cost. }\end{array}$ \\
\hline Investment Recovery & $\begin{array}{l}\text { Provide facilities, trained personnel, services, and management } \\
\text { controls for the timely identification and disposition of assets } \\
\text { excess to the needs of the site through redeployment, recycling } \\
\text { initiatives, and public sale of surplus property. }\end{array}$ \\
\hline Property Systems & $\begin{array}{l}\text { Operate, maintain and enhance the development of cost effective } \\
\text { data systems to control and adninister multi-contractor personal } \\
\text { property assets. }\end{array}$ \\
\hline
\end{tabular}




\begin{tabular}{|c|c|c|c|c|c|}
\hline \multicolumn{3}{|c|}{ 2.B.1 Milestone List } & \multicolumn{2}{|c|}{$\begin{array}{c}\text { Westinghouse Hanford Company } \\
\text { Prograin Title } \\
\text { SMS/WBS No. } 6.10 .7 \\
\end{array}$} & $\begin{array}{l}\text { FY } 1995 \\
\text { Site Support Program Plan } \\
\text { Date Prepared: } 06 / 28 / 94 \\
\end{array}$ \\
\hline \multicolumn{2}{|c|}{ Milestone } & \multirow{2}{*}{$\begin{array}{l}\text { WBS } \\
\text { Number }\end{array}$} & \multirow{2}{*}{\multicolumn{2}{|c|}{ Milestone Description }} & \multirow{2}{*}{$\begin{array}{l}\text { Due } \\
\text { Date }\end{array}$} \\
\hline Type & Number & & & & \\
\hline RL & $\begin{array}{l}\text { MM0-95- } \\
001\end{array}$ & 6.10 .7 & $\begin{array}{l}\text { Sustain "0" Lost Work Day } \\
\text { injuries in Materials } \\
\text { Management in FY } 1995 .\end{array}$ & & $09 / 30 / 95$ \\
\hline RL & $\begin{array}{l}\text { MMO-95- } \\
002\end{array}$ & 6.10 .7 & $\begin{array}{l}\text { Develop and implement a } \\
\text { site wide training } \\
\text { program for contractor } \\
\text { employees on the } \\
\text { Utilization and Disposal } \\
\text { of Excess Property. }\end{array}$ & & $12 / 31 / 94$ \\
\hline $\mathrm{RL}$ & $\begin{array}{l}\text { PMR-95- } \\
001 \\
\text { through } \\
\text { PMR-94- } \\
004\end{array}$ & 6.10 .7 .1 & $\begin{array}{l}\text { Complete } 1 / 4 \text { of required } \\
\text { tagged property } \\
\text { inventories. }\end{array}$ & & $\begin{array}{l}12 / 31 / 94 \\
03 / 31 / 95 \\
06 / 30 / 95 \\
09 / 30 / 95\end{array}$ \\
\hline RL & $\begin{array}{l}\text { PMR-95- } \\
005 \\
\text { through } \\
\text { PMR-95- } \\
008\end{array}$ & 6.10 .7 .1 & $\begin{array}{l}\text { Complete } 1 / 4 \text { of required } \\
\text { materials inventories. }\end{array}$ & & $\begin{array}{l}12 / 31 / 94 \\
03 / 31 / 95 \\
06 / 30 / 95 \\
09 / 30 / 95\end{array}$ \\
\hline RL & $\begin{array}{l}\text { PMR-95- } \\
009 \text { and } \\
\text { PMR-95- } \\
010\end{array}$ & 6.10 .7 .1 & $\begin{array}{l}\text { Maintain a cumulative } \\
\text { inventory accuracy rate } \\
\text { of at least } 97 \% \text {. Status } \\
\text { to be reported semi- } \\
\text { annually. }\end{array}$ & & $\begin{array}{l}03 / 31 / 95 \\
09 / 30 / 95\end{array}$ \\
\hline
\end{tabular}




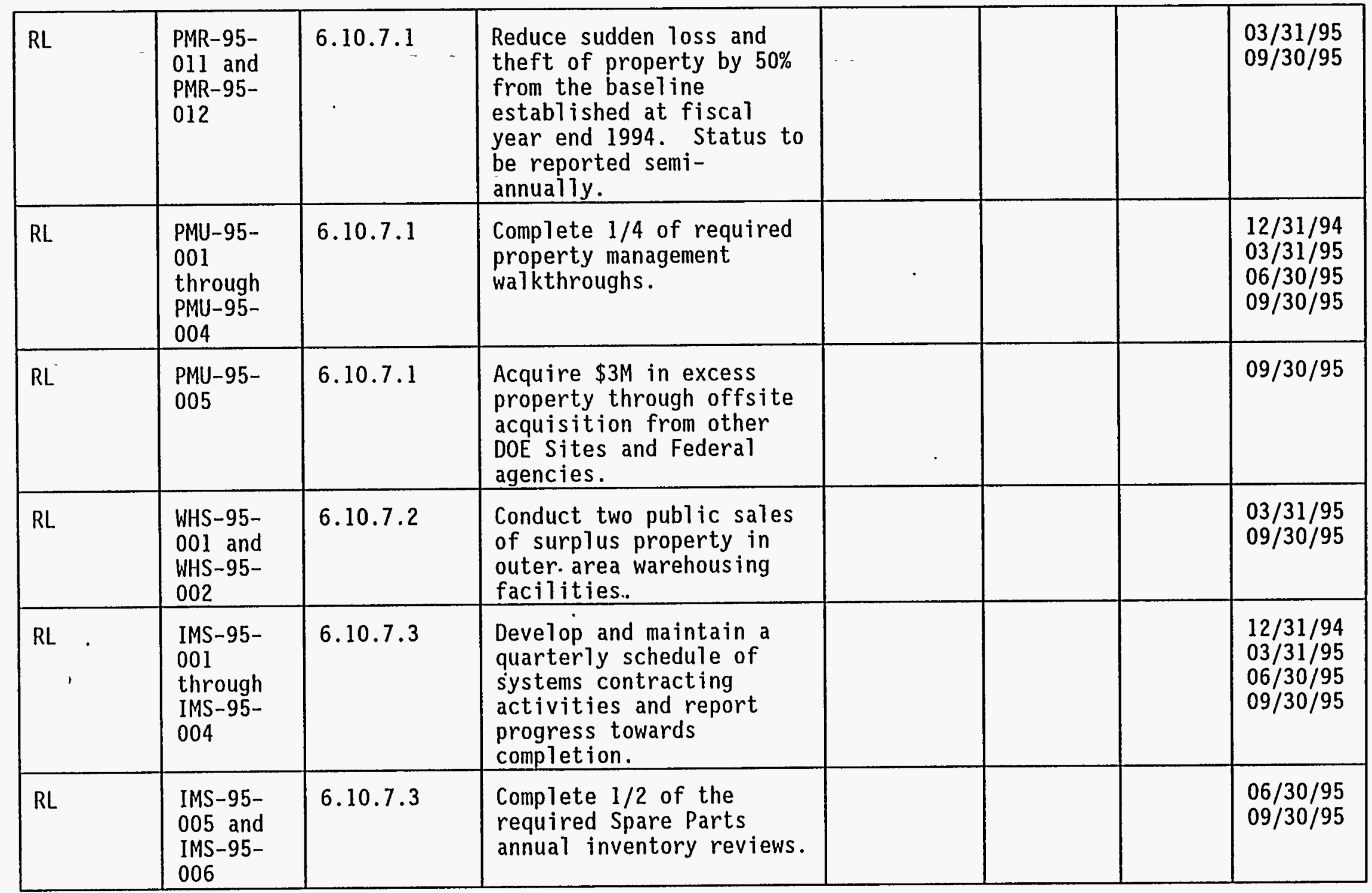




\begin{tabular}{|c|c|c|c|c|}
\hline $\mathrm{RL}$ & $\begin{array}{l}\text { IMS-95- } \\
007 \cdot \text { and } \\
\text { IMS-95- } \\
008\end{array}$ & 6.10 .7 .3 & $\begin{array}{l}\text { Perform continuous } \\
\text { reviews and } \\
\text { rejustifications of } \\
\text { convenience storage } \\
\text { inventory and report } \\
\text { progress semi-annually. }\end{array}$ & $\begin{array}{l}03 / 31 / 95 \\
09 / 30 / 95\end{array}$ \\
\hline RL & $\begin{array}{l}\text { IMS-95- } \\
009\end{array}$ & 6.10 .7 .3 & $\begin{array}{l}\text { Reduce inventory line } \\
\text { items by } 20 \% \text {, and } \\
\text { inventory value by } 25 \% \text {, } \\
\text { of the ending balance } \\
\text { from FY } 1994 \text { through } \\
\text { aggressive ARDT actions } \\
\text { and through Systems } \\
\text { Contracting. }\end{array}$ & $09 / 30 / 95$ \\
\hline$R L$ & $\begin{array}{l}\text { IRO-95- } \\
001 \\
\text { through } \\
\text { IR0-95- } \\
004\end{array}$ & 6.10 .7 .4 & $\begin{array}{l}\text { Conduct four public } \\
\text { auctions of surplus } \\
\text { property. }\end{array}$ & $\begin{array}{l}12 / 31 / 94 \\
03 / 31 / 95 \\
06 / 30 / 95 \\
09 / 30 / 95\end{array}$ \\
\hline RL & $\begin{array}{l}\text { IR0-95- } \\
005 \\
\text { through } \\
\text { IR0-95- } \\
008\end{array}$ & 6.10 .7 .4 & $\begin{array}{l}\text { Develop and maintain a } \\
\text { quarterly schedule of } \\
\text { Asset Redeployment } \\
\text { Disposal Team (ARDT) } \\
\text { activities and report } \\
\text { completion of tasks by } \\
\text { facility and area. }\end{array}$ & $\begin{array}{l}12 / 31 / 94 \\
03 / 31 / 95 \\
06 / 30 / 95 \\
09 / 30 / 95\end{array}$ \\
\hline $\mathrm{RL}$ & $\begin{array}{l}\text { IR0-95- } \\
009 \text { and } \\
\text { IR0-95- } \\
010\end{array}$ & 6.10 .7 .4 & $\begin{array}{l}\text { Publish a semi-annual } \\
\text { report of Recycling } \\
\text { accomplishments and } \\
\text { initiatives. }\end{array}$ & $\begin{array}{l}03 / 31 / 95 \\
09 / 30 / 95\end{array}$ \\
\hline
\end{tabular}

Materials Management Page 30 


\begin{tabular}{|l|c|c|}
\hline 2.C.1.1 & Westinghouse Hanford Company & FY 1995 SSPP \\
Cost Baseline by & Materials Management & Date Prepared: \\
Activity & SMS/WBS No.6.10.7 & $06 / 28 / 94$ \\
\hline
\end{tabular}

FY 1994 Cost Baseline (Dollars in Thousands)

\begin{tabular}{|l|l|l|}
\hline Activity & \multicolumn{1}{|c|}{ Description } & Total \$s \\
\hline 6.10 .7 .1 & Property Management & $\$ 2,716$ \\
\hline 6.10 .7 .2 & Hanford Asset Recovery/Material Control & $\$ 2,240$ \\
\hline 6.10 .7 .3 & Central Warehouse Operations & $\$ 4,713$ \\
\hline 6.10 .7 .4 & Inventory Control & $\$ 1,110$ \\
\hline & {[} & \\
\hline & {$[$ Excludes company adders (G\&A and CSP/Oversight)] FY 1994 Total for (SMS/WBS No) } & $\$ 11,154$ \\
\hline
\end{tabular}

FY 1995 Cost Baseline (Dollars in Thousands)

\begin{tabular}{|l|l|l|}
\hline 6.10 .7 .0 & Materials Management Manager's Office & $\$$ \\
\hline 6.10 .7 .1 & Property Management & $\$ 1,922$ \\
\hline 6.10 .7 .2 & Warehousing & $\$ 5,905.8$ \\
\hline 6.10 .7 .3 & Inventory Management & $\$ 1,718.9$ \\
\hline 6.10 .7 .4 & Investment Recovery & $\$$ \\
\hline 6.10 .7 .5 & Property Systems & 503 \\
\hline & {$[$ Excludes company adders (G\&A and CSP/Oversight)] FY 1995. Total for (SMS/WBS No) } & $\$ 10,867.7$ \\
\hline
\end{tabular}

Materials Management Page 31 


\begin{tabular}{|l|c|c|}
\hline $\begin{array}{l}\text { 2.C.1.1 } \\
\text { Cost Baseline by } \\
\text { Activity }\end{array}$ & Westinghouse Ilanford Company & FY 1995 SSPP \\
Date Prepared: \\
$06 / 28 / 91$
\end{tabular}

FY 1996 Cost Baseline (Dollars in Thousands)

\begin{tabular}{|l|l|l|}
\hline Activity & \multicolumn{1}{|c|}{ Description } & Total $\$$ s \\
\hline 6.10 .7 & Materials Management Manager's Office & $\$ 232.8$ \\
\hline 6.10 .7 .1 & Property Management & $\$ 1.979 .6$ \\
\hline 6.10 .7 .2 & Warehousing & $\$ 6,083$ \\
\hline 6.10 .7 .3 & Inventory Manayement & $\$ 1,770.5$ \\
\hline 6.10 .7 .1 & Investinent Recovery & $\$ 18$ \\
\hline 6.10 .7 .5 & Property Systems & $\$ 109.8$ \\
\hline & [Excludes company adders (G\&A and CSP/Oversight)] FY 1996 Total for (SHS/WBS No) & $\$ 11,193.7$ \\
\hline
\end{tabular}

Fy 1997 Cost Baseline (Dollars in Thousands)

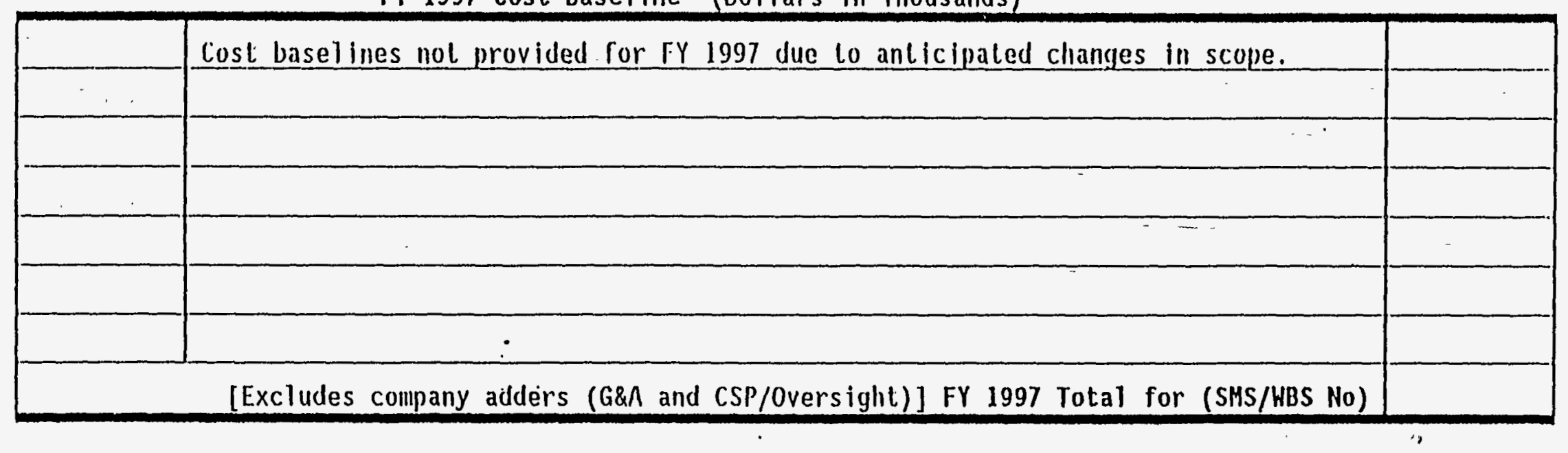




\begin{tabular}{|l|c|c|}
\hline 2.C.2 Cost Basis & $\begin{array}{c}\text { Westinghouse Ilanford Company } \\
\text { Program Title } \\
\text { SMS/WBS No.6.10.7 }\end{array}$ & $\begin{array}{c}\text { FY } 1995 \\
\text { Site Support Program P1an } \\
\text { Date Prepared } \\
06 / 28 / 94\end{array}$ \\
\hline
\end{tabular}

N11 staff are level of effort with the exception of 17.5 non-aligned direct FTE!s. The average company rates used are for each category of employee at 1,812 realization hours for FY-1995.

Assessments are based on current year rates. Other expenses are based on average historical needs as well as current information where available.

Escalation rale from WIIC Controller guidance is $3 \%$. 


\begin{tabular}{|c|c|c|c|c|c|c|c|c|}
\hline \multicolumn{4}{|c|}{ Ful1-Time Equivalent Staff by Job Description } & \multicolumn{5}{|c|}{ NOTE: Job Family only nfter 1996} \\
\hline 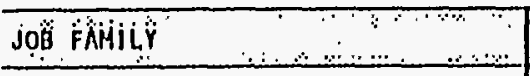 & के & 1: & and & ४०: & +3:3 & +ి० & 3 & \\
\hline Job cntegory & 1994 & 1995 & 1996 & 1997 & 1990 & 1999 & 2000 & 2001 \\
\hline Hiniposs & $14+$ & 15 & 15 & $\because$ & $\because \quad \square$ & $\because$ & $\therefore \quad: \quad 3$ & \\
\hline first line & 12 & 13 & 13 & & & & & \\
\hline Genernl/executive & 2 & 2 & 2 & & & & & \\
\hline \multicolumn{9}{|l|}{ Project/rrogran } \\
\hline \multicolumn{9}{|l|}{ other } \\
\hline \multicolumn{9}{|l|}{ EIIGjIIEEERS } \\
\hline \multicolumn{9}{|l|}{ Chemical } \\
\hline \multicolumn{9}{|l|}{ civil } \\
\hline \multicolumn{9}{|l|}{ Conputer } \\
\hline \multicolumn{9}{|l|}{ Electrical } \\
\hline \multicolumn{9}{|l|}{ Envirornnental } \\
\hline \multicolumn{9}{|l|}{ Industrial } \\
\hline \multicolumn{9}{|l|}{ Mechnnicnl } \\
\hline \multicolumn{9}{|l|}{ Nuclear } \\
\hline \multicolumn{9}{|l|}{ Petroleum/Mining } \\
\hline \multicolumn{9}{|l|}{ Plant } \\
\hline \multicolumn{9}{|l|}{ auallity control } \\
\hline \multicolumn{9}{|l|}{ Sofety } \\
\hline \multicolumn{9}{|l|}{ otlier } \\
\hline 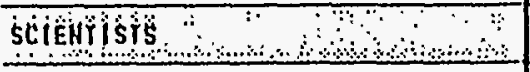 & 12+ & 2 & 3 & 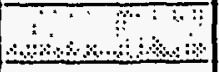 & $2 \times 3$ & $2+3$ & 8 & \\
\hline \multicolumn{9}{|c|}{\begin{tabular}{|l|l|l|l|l|l|} 
Chemists & & & & & \\
\end{tabular}} \\
\hline \multicolumn{9}{|l|}{ Envirommental } \\
\hline \multicolumn{9}{|l|}{ Geologists } \\
\hline \multicolumn{9}{|l|}{ Life } \\
\hline Haterial & & & & & & & & \\
\hline
\end{tabular}




\begin{tabular}{|c|c|c|c|c|c|c|c|c|}
\hline Ful1-Time Equivalent Staf & $f$ by Job $D$ & escription & & NOTE: Jo & b Family & Only nfte & er 1996 & \\
\hline Mathematicians & & $\cdot$ & & & & & & \\
\hline Physicists & & & & & & & & \\
\hline Social & & & & & & & & \\
\hline other & & & & & & & & \\
\hline 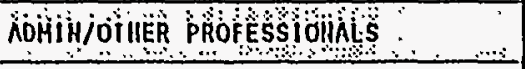 & $39: 8 \div \div$ & $38 \div$ & 38.5 & $\eta$ & & $\therefore \times \cdots$ & & $\because$ \\
\hline Accountant/auditor &. & & & & & & & \\
\hline Architect & & & & & & & & \\
\hline Buyers/procurement & & & & & & & & \\
\hline Conmminications & & & & & & & & \\
\hline Compliance inspectors & & & & $\cdot$ & & & & \\
\hline Conputer System Anal & & & & & & & & \\
\hline Cost Est/plonmer/sch & & & & & & & & \\
\hline Heal th Physics & & & & & & & & \\
\hline lixluste'inl Hygiene & & & & & & & & \\
\hline Lahyers & & & & & & & & \\
\hline Personnel/Lnbor Rela & & & & & & & & \\
\hline Pliysicians & & & & & & & & \\
\hline Physician Assis/liurs & & $\dot{-}$ & & & & & & \\
\hline Safeguard \& Security & & & & & & & & \\
\hline Tech Hriters \& Edit & & & & & & & & \\
\hline Irainers & & & & & & & & \\
\hline other & 39.8 & 38.5 & 38.5 & & & & & \\
\hline jos kififlu & अ०० & $3 \times$ & 6 & and & $\therefore$ & B... & 4 & \\
\hline Job category & & & & & & & & \\
\hline 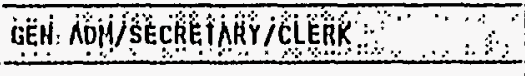 & 335 & 3000 & 3000 & $\because \because \quad 3$ & आय & $0<\therefore$ & $\therefore \quad \cdots$ & 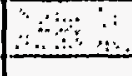 \\
\hline Actuin Assistnints & & & & & & & & \\
\hline office rilerks (Gen) & 33.5 & 30.0 & 30.0 & & & & & \\
\hline orfice clerks (specinl) & & & & & & & & \\
\hline
\end{tabular}


Ful1-Time Equivalent Staff by Job Description

\begin{tabular}{|c|c|c|}
\hline \multicolumn{3}{|l|}{ Secretarles } \\
\hline \multicolumn{3}{|l|}{ Typist/Hord Process } \\
\hline \multicolumn{3}{|l|}{ other } \\
\hline \multicolumn{3}{|l|}{ iEçiNiciAlls $\ldots$} \\
\hline \multicolumn{3}{|l|}{ Computer Oper/Coder } \\
\hline \multicolumn{3}{|l|}{ orafters } \\
\hline \multicolumn{3}{|l|}{ Engrs/Tech } \\
\hline \multicolumn{3}{|l|}{ Envir. Scl Techuicians } \\
\hline \multicolumn{3}{|l|}{ Nealth Phys. Technic. } \\
\hline \multicolumn{3}{|l|}{ Iindus. Sof/llealth Jech } \\
\hline \multicolumn{3}{|l|}{ Instru/Control lech } \\
\hline \multicolumn{3}{|l|}{ Lob. Technicians } \\
\hline \multicolumn{3}{|l|}{ Medin Teclinicians } \\
\hline \multicolumn{3}{|l|}{ Survey/Map Iech } \\
\hline \multicolumn{3}{|l|}{ otlier } \\
\hline cRhifts & s.mon & : \\
\hline \multicolumn{3}{|c|}{\begin{tabular}{|l|l|l|} 
Carpenters & \\
\end{tabular}} \\
\hline \multicolumn{3}{|l|}{ Electricians } \\
\hline \multicolumn{3}{|l|}{ IIVnC } \\
\hline \multicolumn{3}{|l|}{ Machinists } \\
\hline \multicolumn{3}{|l|}{ Masons } \\
\hline \multicolumn{3}{|l|}{ Mlllurights } \\
\hline \multicolumn{3}{|l|}{ Palnters } \\
\hline \multicolumn{3}{|l|}{ Plumers/pipefitters } \\
\hline \multicolumn{3}{|l|}{ Struct/Metal Workers } \\
\hline \multicolumn{3}{|l|}{ Vehic./Mob Equip Mech } \\
\hline \multicolumn{3}{|l|}{ Helders } \\
\hline other & & \\
\hline
\end{tabular}

\begin{tabular}{|c|c|c|c|c|}
\hline \multicolumn{2}{|c|}{ NOTE: Job Family } & \multicolumn{2}{|c|}{ Only nfter 1.996} & \multirow{2}{*}{ 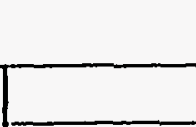 } \\
\hline & & & & \\
\hline & & & & \\
\hline & & & & \\
\hline & & : & $\therefore \%, \ldots$ & \\
\hline & & & & \\
\hline & & & & \\
\hline & & & & \\
\hline & & & & \\
\hline & & & - & \\
\hline & & & & \\
\hline & & & & \\
\hline & & & & \\
\hline & & & & \\
\hline & & & & \\
\hline & & & & \\
\hline & ? & $\square$ & $\because \quad \therefore$ & . \\
\hline & & & & - \\
\hline & & & & \\
\hline & & & & \\
\hline & & & & \\
\hline & & & & - \\
\hline & & & & \\
\hline & & & . & \\
\hline & & & & .... \\
\hline & & & - & \\
\hline & & & & \\
\hline & & & & \\
\hline & & & 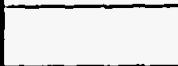 & \\
\hline
\end{tabular}




\begin{tabular}{|c|c|c|c|c|c|c|c|c|}
\hline \multicolumn{4}{|c|}{ Full-Time Equivalent Staff by Job Description } & \multicolumn{5}{|c|}{ NOTE: Job Family only After 1996} \\
\hline opERNónS 134 & $\therefore$ & $8+4$ & 3 & अ०: & 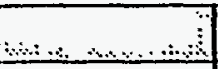 & 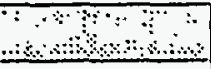 & & \\
\hline Chemlcal system & & & & & & $\cdot$ & & \\
\hline Drillers & & & & & & & & \\
\hline Lt. Vehicle Driver & & & & & & & & \\
\hline Materinl Moving En & & & & & & & & \\
\hline Nuclear Plant & & & & & & & & \\
\hline Utilities Haste $\mathrm{Pr}$ & & & & & & & & \\
\hline other & & & & & & & & \\
\hline LABOR \& GEHUOORKERS & 80 & 870 & נ艹 & & & & . & \\
\hline Firefighters & & & & & & & & \\
\hline Food Service & & & & & & & & \\
\hline Hand/Help Lab Gen & & & & & & & & \\
\hline Hand/Help Lab spec & & & & & & & & \\
\hline Janitors/Cleoners & & & & & & & & \\
\hline Loundry Horkers & & & & & & & & \\
\hline Security Guards & & & & & r & & & \\
\hline other & 87.0 & 87.0 & 87.0 & & & & & \\
\hline
\end{tabular}




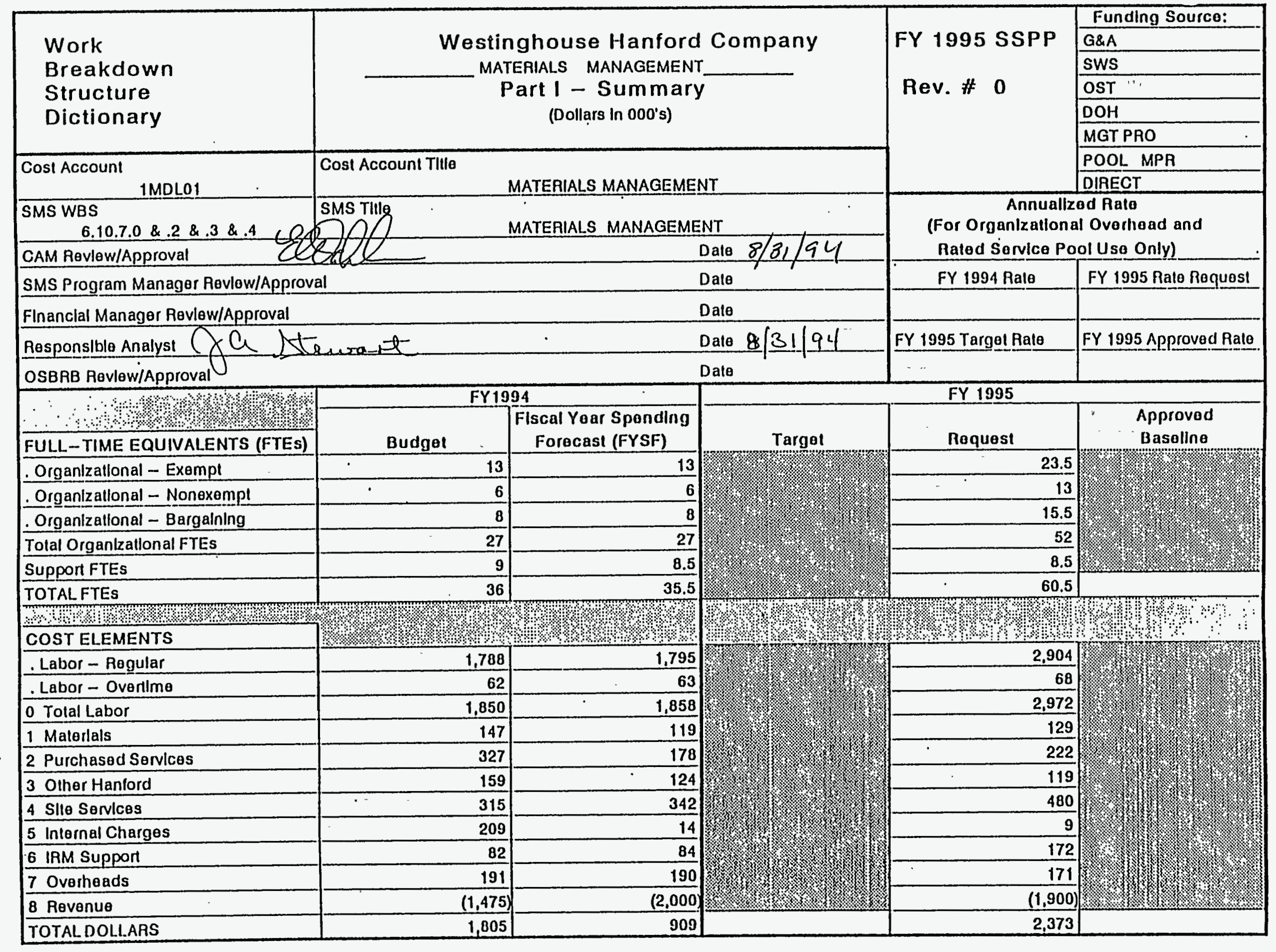




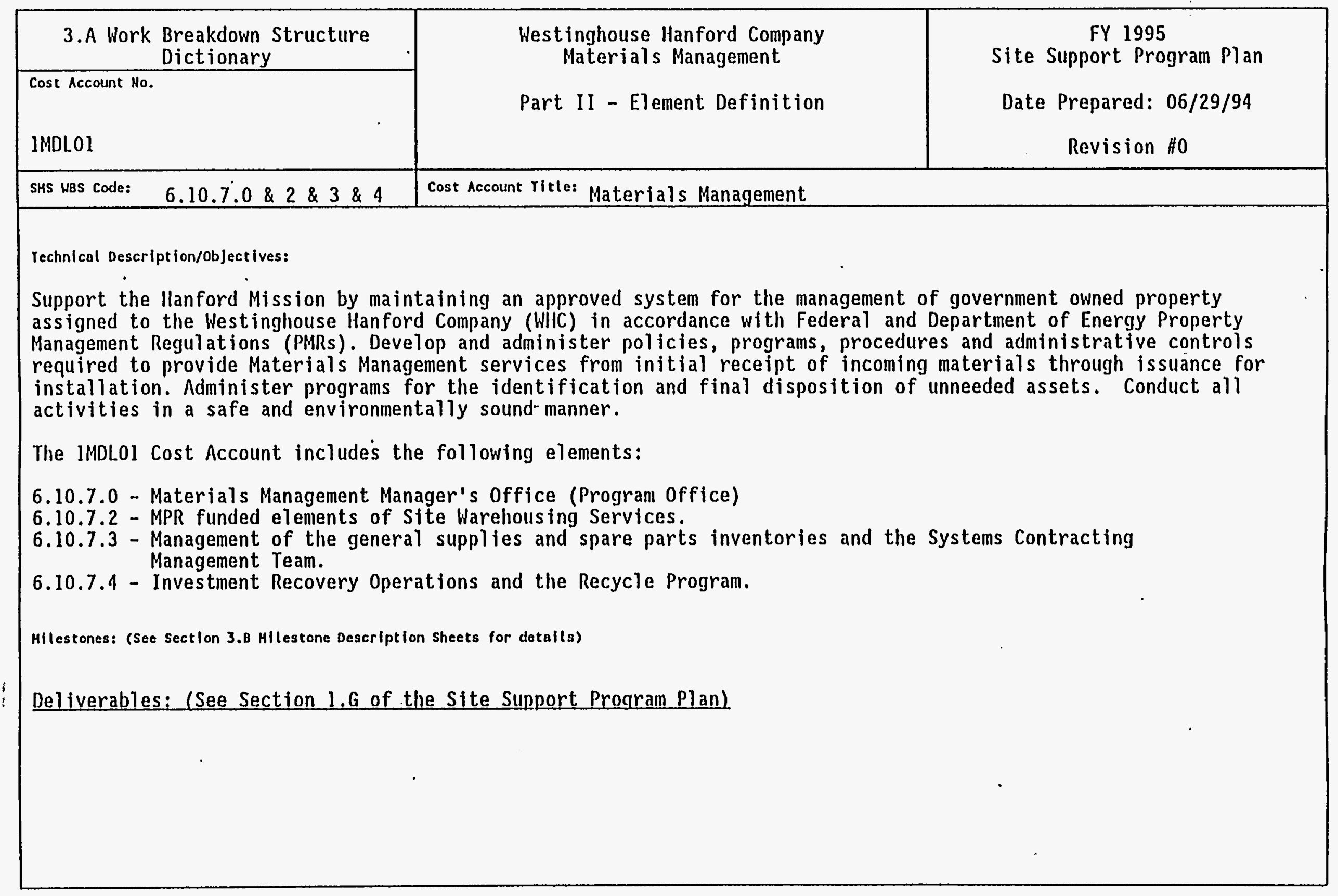




\begin{tabular}{|c|c|c|c|c|c|c|c|c|c|c|}
\hline Cost Account Ho. $1 M D L 01$ & \multicolumn{6}{|c|}{ Part II - Materials Management } & \multicolumn{4}{|c|}{6.10 .7 .0} \\
\hline Element Task Description & \multicolumn{2}{|c|}{1994 FYSF } & \multicolumn{2}{|c|}{ Delta } & \multicolumn{2}{|c|}{ FY 1995} & \multicolumn{2}{|c|}{ Delta } & \multicolumn{2}{|c|}{ FY 1996} \\
\hline Requests Within Target: & FTEs & $\$ s$ & FTES & $\$ s$ & FTES & $\$ s$ & FTES & $\$ s$ & FTES & $\$ s$ \\
\hline $\begin{array}{l}\text { Activity Detailed Description: } \\
\text { 6.10.7.0 Materials Management Manager's } \\
\text { Office (Program Office) } \\
\text { Provide Management overview and } \\
\text { administrative support for the Materials } \\
\text { Management Program. Monitor organizational } \\
\text { performance against Site Support Program } \\
\text { Plan objectives, deliverables and } \\
\text { milestones. }\end{array}$ & 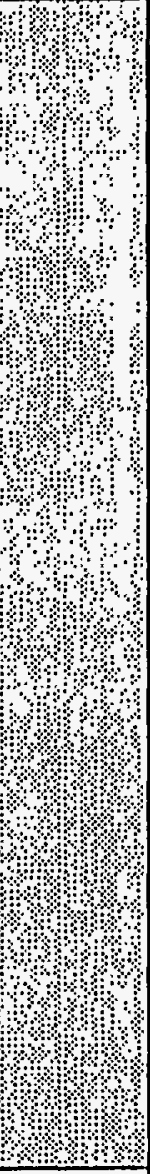 & 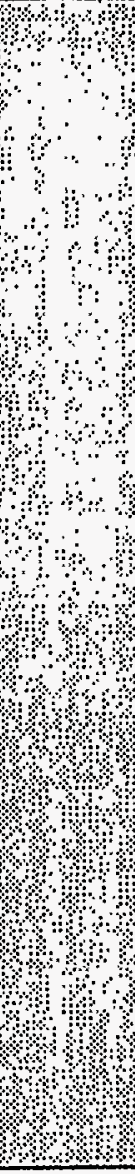 & 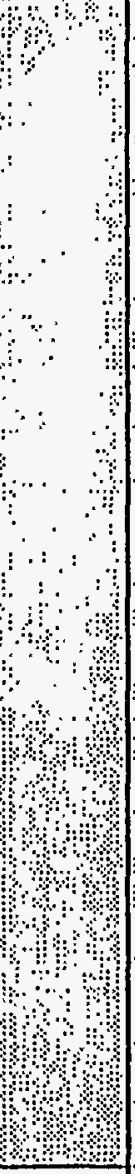 & 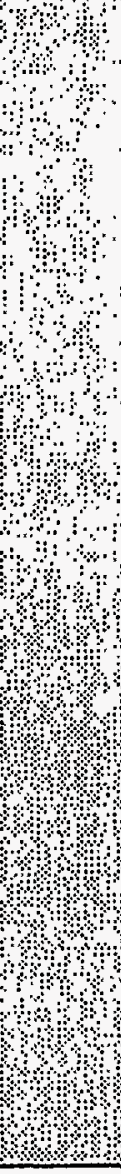 & 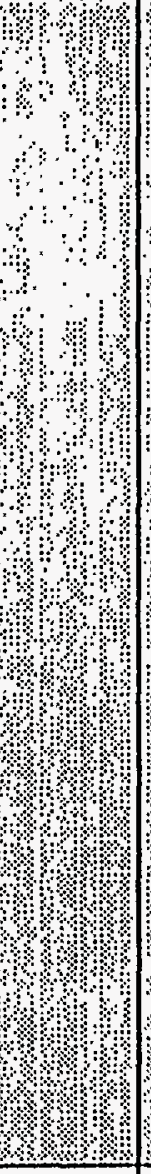 & 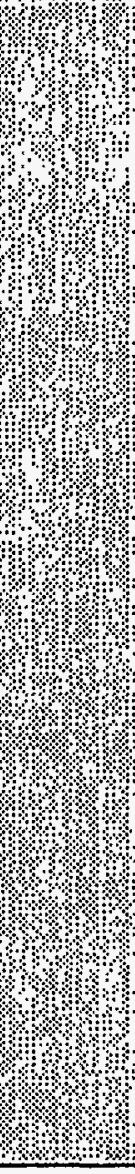 & 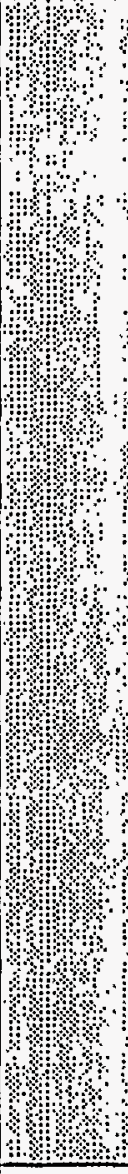 & 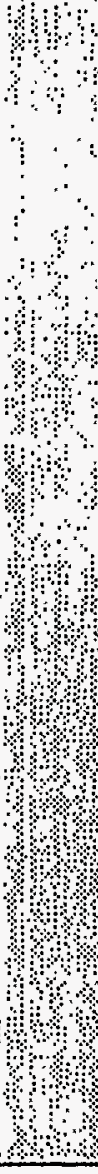 & 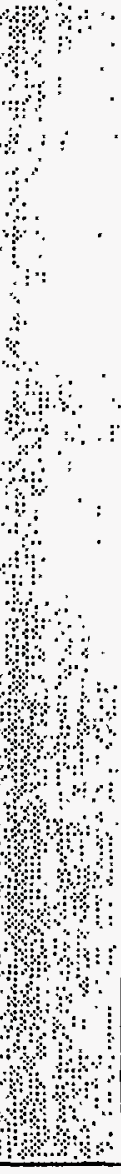 & 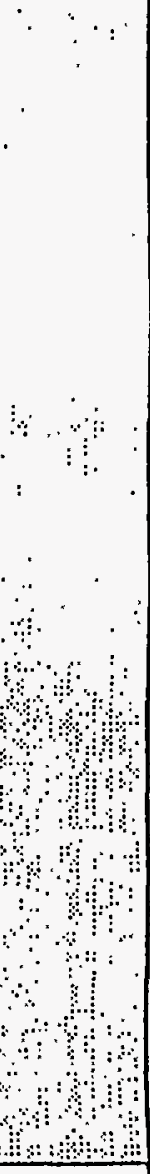 \\
\hline 6.10.7.0 Labor & $N / A$ & $N / A$ & $N / A$ & $N / A$ & 2.0 & 89.1 & & & 2.0 & 91.8 \\
\hline 6.10.7.0 Non-Labor & $N / A$ & $N / A$ & $N / A$ & $N / A$ & $N / A$ & 136.9 & & & 2.0 & 141.0 \\
\hline
\end{tabular}




\begin{tabular}{|c|c|c|c|c|c|c|c|c|c|c|}
\hline Cost Account No. $1 M D L 01$ & \multicolumn{6}{|c|}{ Part II - Materials Management } & \multicolumn{4}{|c|}{6.10 .7 .2} \\
\hline Element Task Description & \multicolumn{2}{|c|}{1994 FYSF } & \multicolumn{2}{|c|}{ Delta } & \multicolumn{2}{|c|}{ FY 1995} & \multicolumn{2}{|c|}{ Delta } & \multicolumn{2}{|c|}{ FY 1996} \\
\hline Requests Within Target: & FTES & $\$ s$ & FTES & $\$ s$ & FTES & $\$ s$ & FTES & $\$$ s & FTES & $\$ s$ \\
\hline $\begin{array}{l}\text { Activity Detailed Description: } \\
6.10 .7 .2 \text { - MPR funded elements of site } \\
\text { Warehousing Services } \\
\text { Provide facilities, trained personnel, } \\
\text { services, and management controls for the } \\
\text { receiving, storage, issuance and preventive } \\
\text { maintenance of spare parts and convenience } \\
\text { storage items stored in outer area } \\
\text { warehousing facilities that support multiple } \\
\text { site programs. }\end{array}$ & 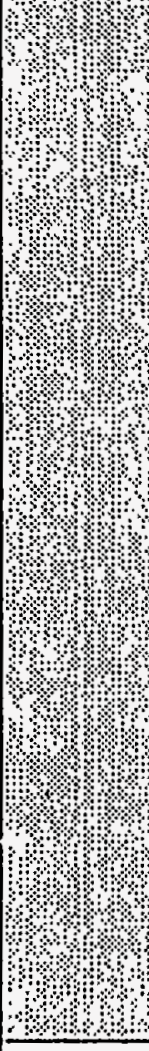 & 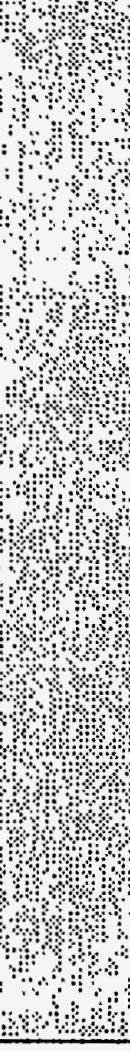 & 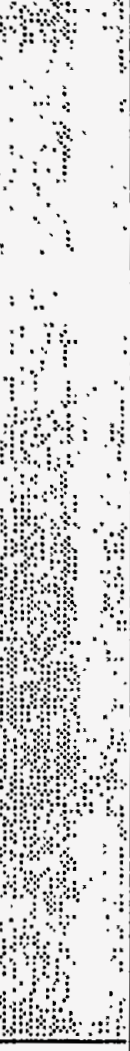 & 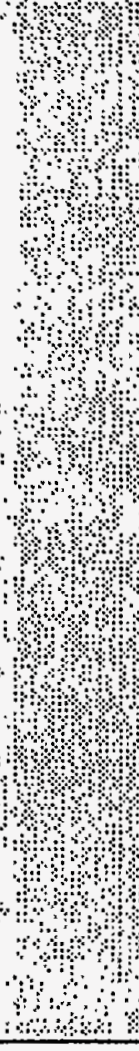 & 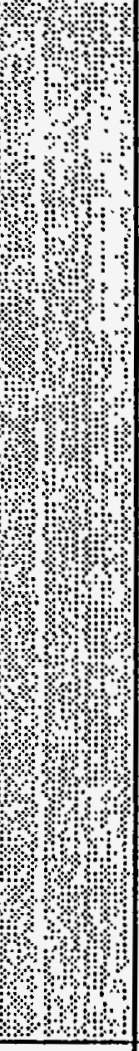 & 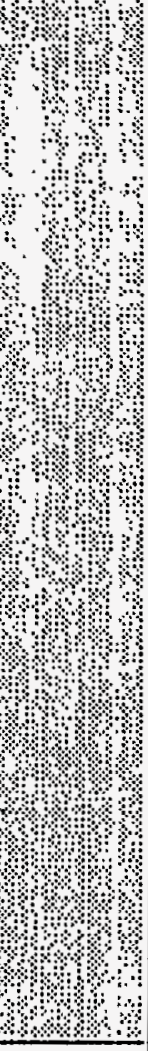 & 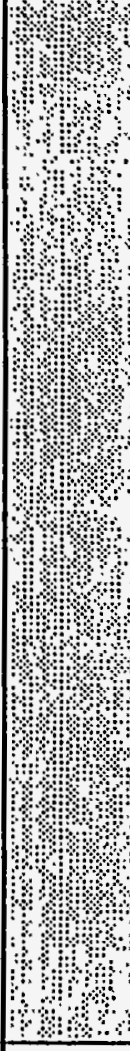 & 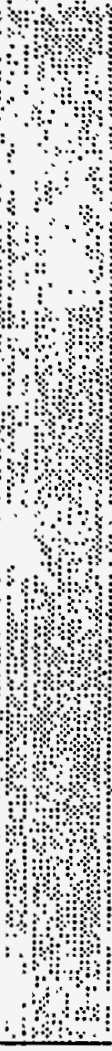 & 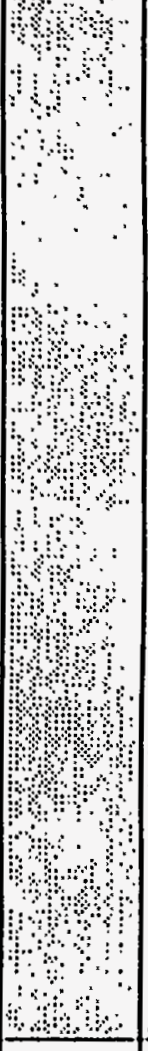 & $\begin{array}{l}\because \\
\because \\
\because \\
\ddots \\
\ddots\end{array}$ \\
\hline 6.10.7.2 Labor & $N / A$ & $N / A$ & $N / \Lambda$ & N/A & 11.0 & 508.6 & & & 11.0 & 523.9 \\
\hline 6.10.7.2 Non-Labor & $N / A$ & $N / A$ & $N / n$ & $N / A$ & N/A & 64.2 & & & $N / \Lambda$ & 66.1 \\
\hline
\end{tabular}




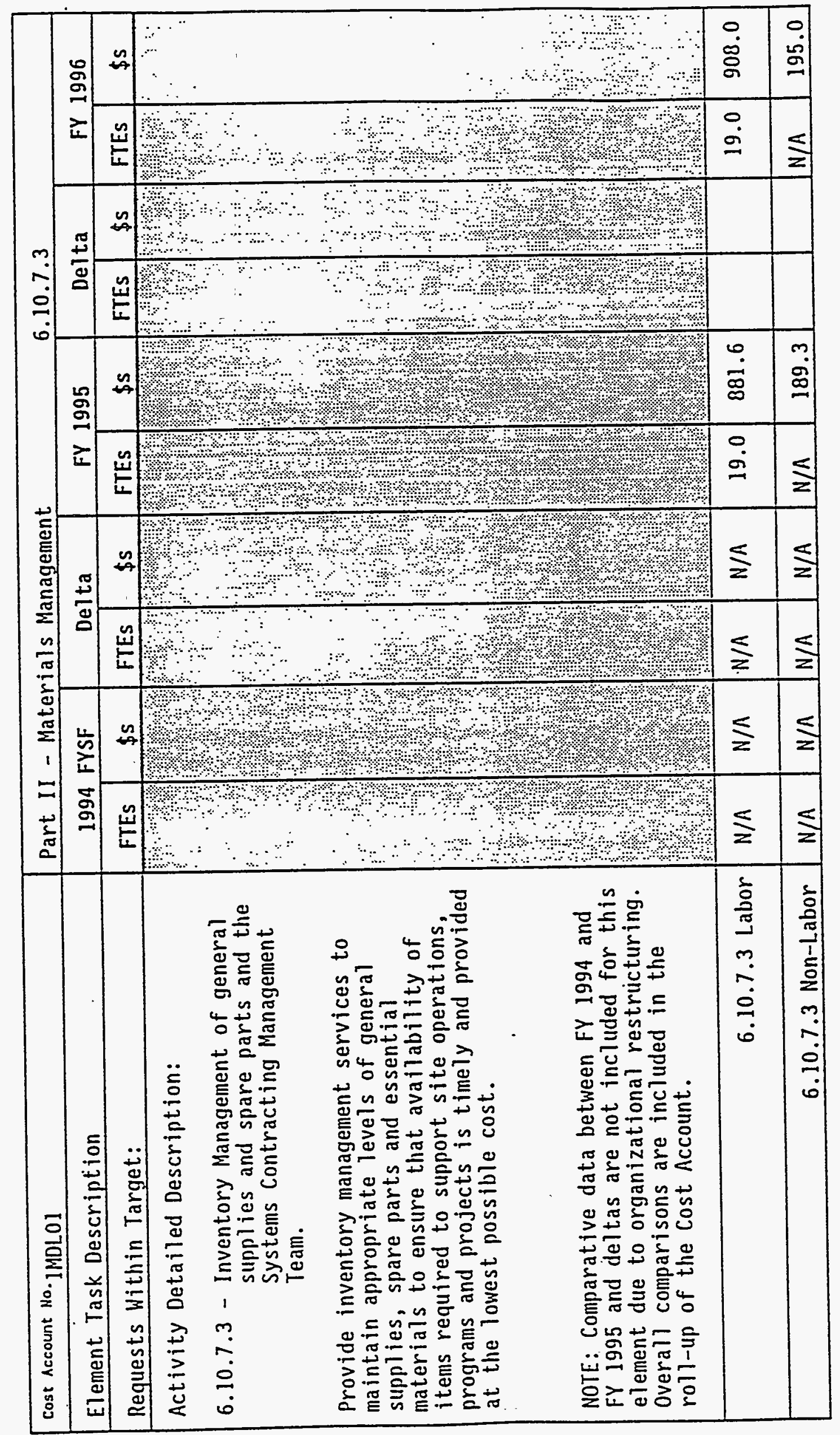

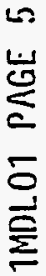




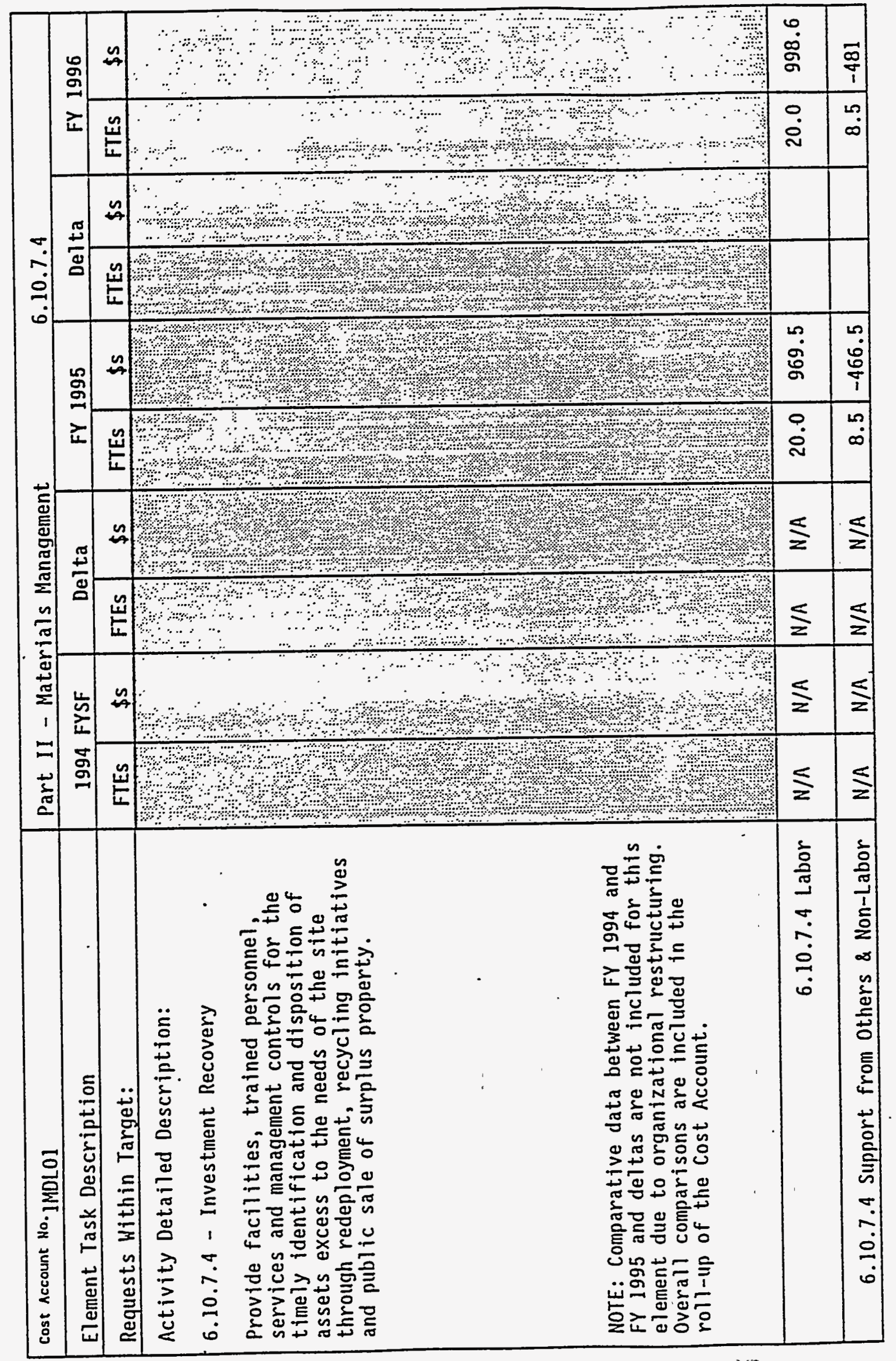

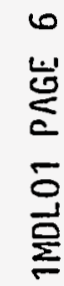




\begin{tabular}{|c|c|c|c|c|c|c|c|c|c|c|}
\hline \multirow{3}{*}{$\begin{array}{l}\text { Cost Account No. } 1 \text { MDLO1 } \\
\text { Element Task Description } \\
\text { Requests Within Target: }\end{array}$} & \multicolumn{6}{|c|}{ Part II - Materials Management } & \multicolumn{4}{|l|}{6.10 .7} \\
\hline & \multicolumn{2}{|c|}{1994 FYSF } & \multicolumn{2}{|c|}{ Delta } & \multicolumn{2}{|c|}{ FY 1995} & \multicolumn{2}{|c|}{ Delta } & \multicolumn{2}{|c|}{ FY 1996} \\
\hline & FTES & $\$ s$ & FTES & $\$ s$ & FTES & $\$ s$ & FTES & $\$ s$ & FTEs & $\$ \mathbf{s}$ \\
\hline $\begin{array}{l}\text { Cost Account roll-up and comparative data } \\
\text { for IMDLO1 }\end{array}$ & 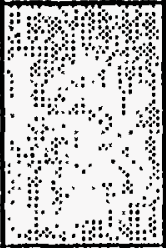 & 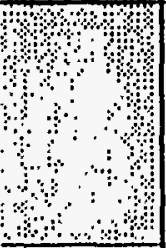 & 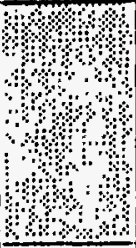 & \begin{tabular}{c}
6 \\
4 \\
\hdashline
\end{tabular} & 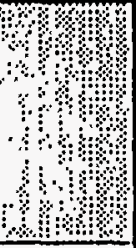 & 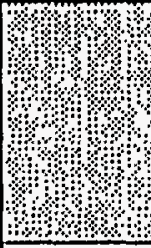 & $\begin{array}{l}0 \% \\
4 \\
3 \\
3\end{array}$ & 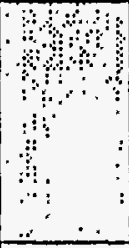 & 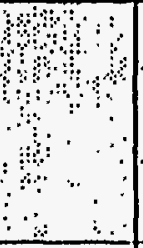 & 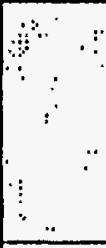 \\
\hline 1MDLO1 Labor & .27 .0 & 1850 & 25.0 & 1114 & 52 & 2972 & 0 & 89 & 52 & 3061 \\
\hline IMDLOi Support from Others and Non-Labor & 9.0 & -45 & -.5 & 350 & 8.5 & -599 & 0 & -18 & 8.5 & 617 \\
\hline 1MDL0l Total Cost Account & 36 & 1805 & 24.5 & 1464 & 60.5 & 2373 & 0 & 71 & 60.5 & 2444 \\
\hline
\end{tabular}




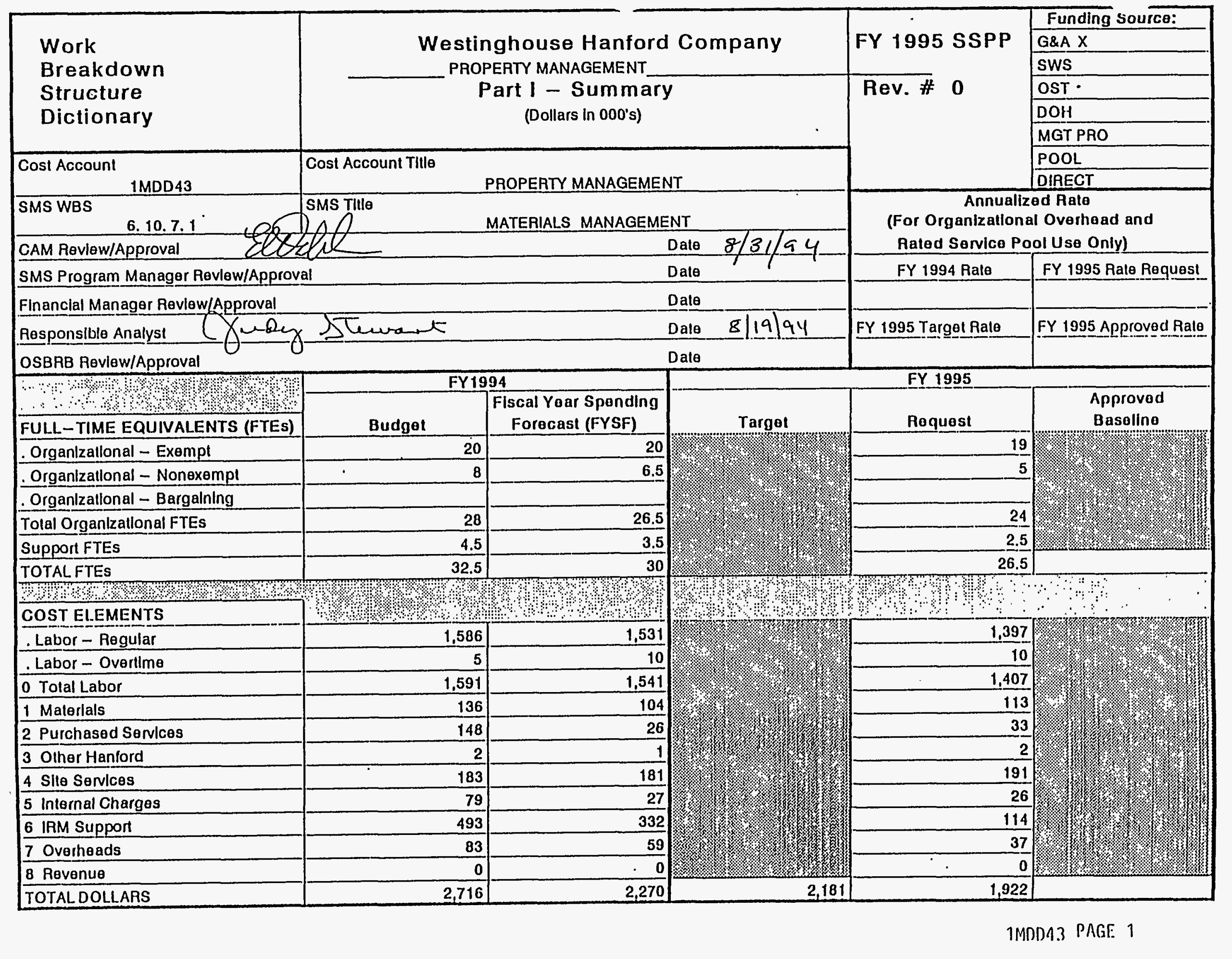




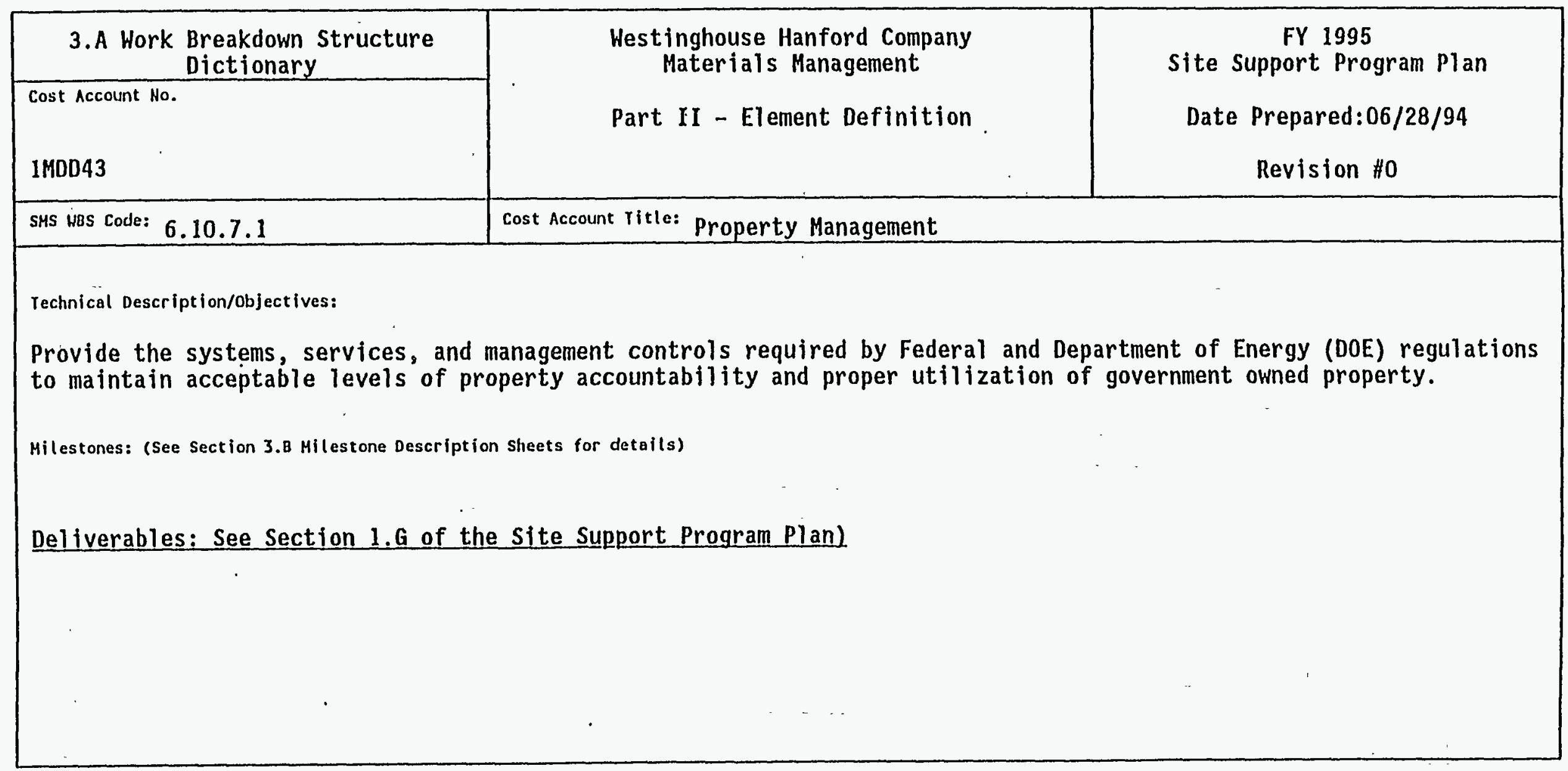




\begin{tabular}{|c|c|c|c|c|c|c|c|c|c|c|}
\hline Cost Account No. 1 MDD43 & \multicolumn{6}{|c|}{ Part II - Materials Management } & \multicolumn{4}{|c|}{6.10 .7 .1} \\
\hline Element Task Description & \multicolumn{2}{|c|}{1994 FYSF } & \multicolumn{2}{|c|}{ Delta } & \multicolumn{2}{|c|}{ FY 1995} & \multicolumn{2}{|c|}{ Delta } & \multicolumn{2}{|c|}{ FY 1996} \\
\hline Requests Within Target: & FTEs & $\$ s$ & FTEs & $\$ S$ & FTEs & $\$ s$ & FTES & $\$ s$ & FTES & $\$ s$ \\
\hline $\begin{array}{l}\text { Activity Detalled Description: } \\
6.10 .7 .1 \text { - Property Management } \\
\text { See page } 3 \text {, Section 1.B.1 of the Site } \\
\text { Support Program Plan }\end{array}$ & ? & 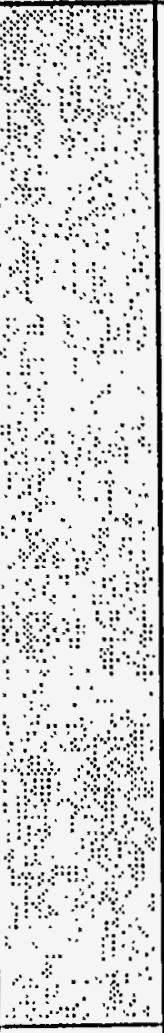 & 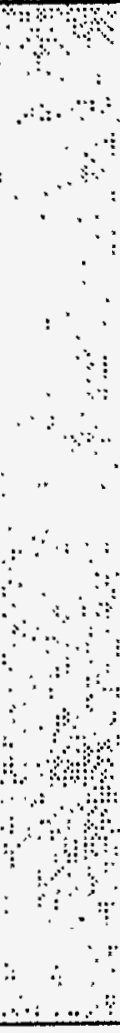 & 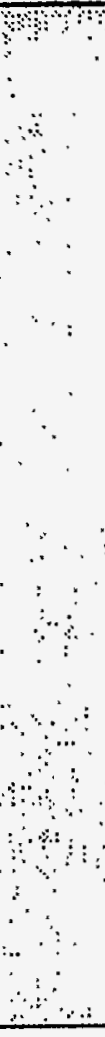 & 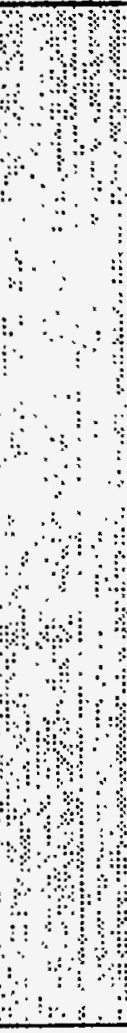 & 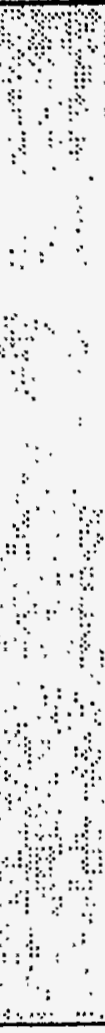 & 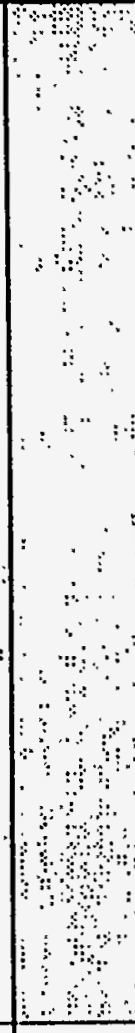 & $\begin{array}{l}\because \\
\because \\
\because \\
\therefore \\
\therefore \quad \\
\therefore \quad \\
\therefore \quad \\
\ddots \\
\vdots \\
\vdots \\
\vdots\end{array}$ & 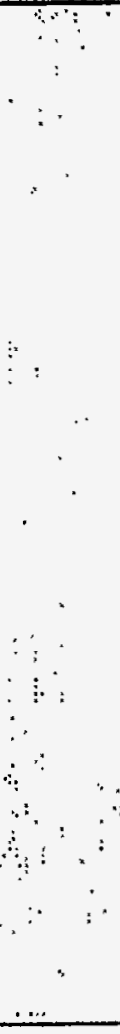 & \\
\hline 1MDD43 Labor & 28 & 1591 & -4 & -184 & 24 & 1407. & 0 & 42.2 & 24 & 1449 \\
\hline 1MDD43 Support From 0thers and Non-Labor & 4.5 & 1125 & -2 & -610 & 2.5 & 515 & 0 & 15.4 & 2.5 & 530.4 \\
\hline 1MDD43 Total Cost Account & 32.5 & 2716 & -6 & -794 & 26.5 & 1922 & 0 & 57.6 & 26.5 & 1979.4 \\
\hline
\end{tabular}




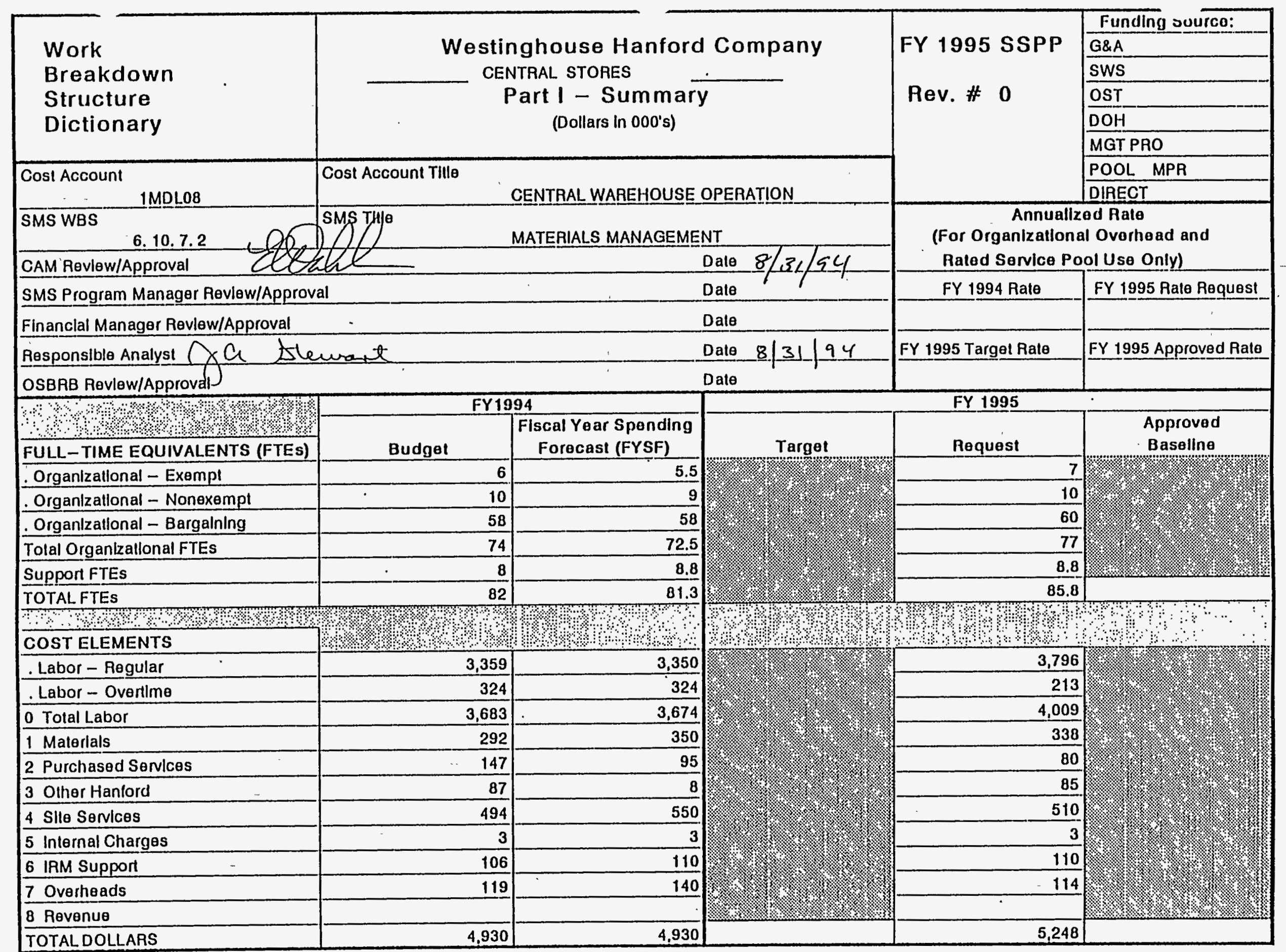




\begin{tabular}{|c|c|c|}
\hline $\begin{array}{l}\text { 3.A Work Breakdown Structure } \\
\text { Dictionary }\end{array}$ & \multirow{3}{*}{$\begin{array}{l}\text { Westinghouse Hanford Company } \\
\text { Materials Management } \\
\text { Part II - Element Definition }\end{array}$} & \multirow{3}{*}{$\begin{array}{c}\text { FY } 1995 \\
\text { Site Support Program Plan } \\
\text { Date Prepared:06/28/94 } \\
\text { Revision } \$ 0\end{array}$} \\
\hline Cost Account No. & & \\
\hline 1MDL08 & & \\
\hline SHS Has code: $\quad 6.10 .7 .2$ & Cost Account Title: Central Warehouse Opera & \\
\hline \multicolumn{3}{|l|}{ Technical Description/objectives: } \\
\hline \multicolumn{3}{|c|}{$\begin{array}{l}\text { Provide facilities, trained personnel, services and management controls for the shipping/receiving, storage, issuanc } \\
\text { and distribution of materials, parts, components and equipment required to support the ongoing operation of the } \\
\text { Hanford Site. } \\
\text { Hilestones: (see section 3.B Hllestone Description sheets for detalls) }\end{array}$} \\
\hline \multicolumn{3}{|c|}{ (See Section 1.G of the Site Support Program Plan) } \\
\hline
\end{tabular}




\begin{tabular}{|c|c|c|c|c|c|c|c|c|c|c|}
\hline Cost Account No.1MDLO8 & \multicolumn{6}{|c|}{ Part II - Materials Management } & \multicolumn{4}{|c|}{6.10 .7 .2} \\
\hline Element Task Description & \multicolumn{2}{|c|}{1994 FYSF } & \multicolumn{2}{|c|}{ De1ta } & \multicolumn{2}{|c|}{ FY 1995} & \multicolumn{2}{|c|}{ Delta } & \multicolumn{2}{|c|}{ FY 1996} \\
\hline Requests Within Target: & FTES & $\$ s$ & FTES & $\$ \mathbf{s}$ & FTES & $\$ s$ & FTES & $\$ s$ & FTEs & $\$ s$ \\
\hline $\begin{array}{l}\text { Activity Detailed Description: } \\
6.10 .7 .2 \text { - Central Warehouse Operations } \\
\text { See page 4, section 1.B.1 of the Site } \\
\text { Support.Program Plan. }\end{array}$ & 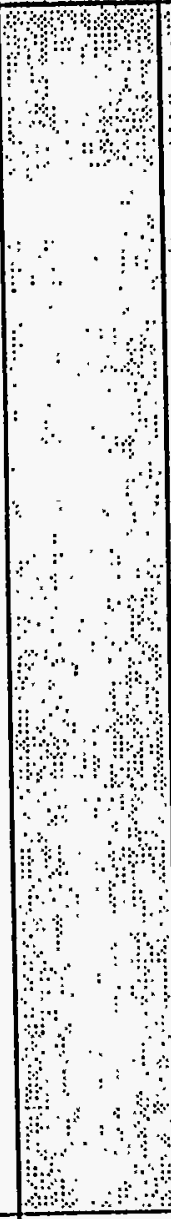 & 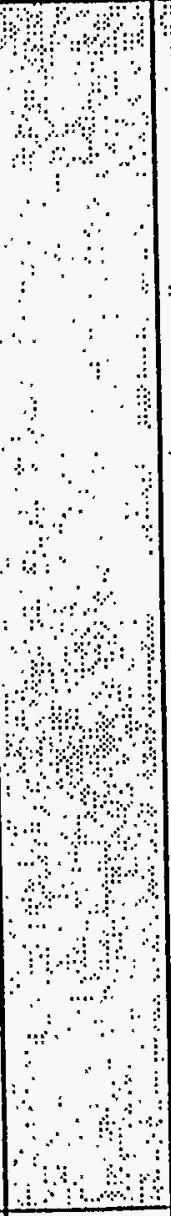 & 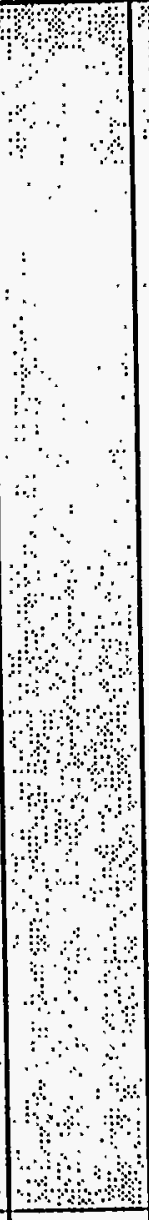 & 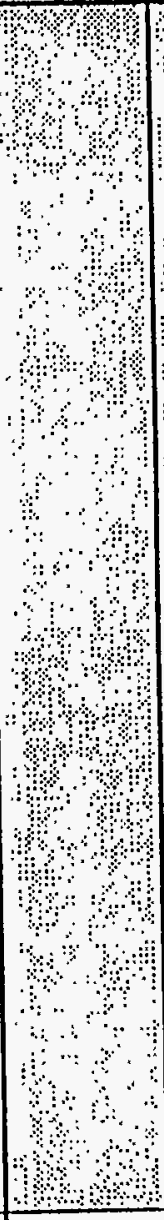 & 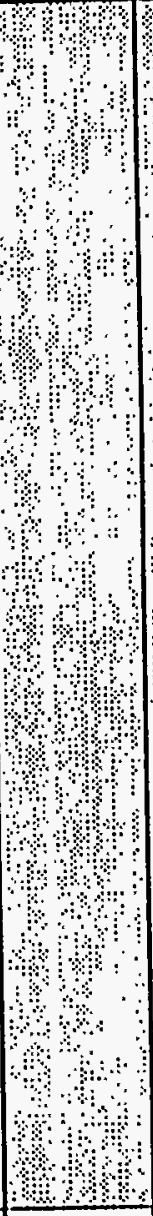 & 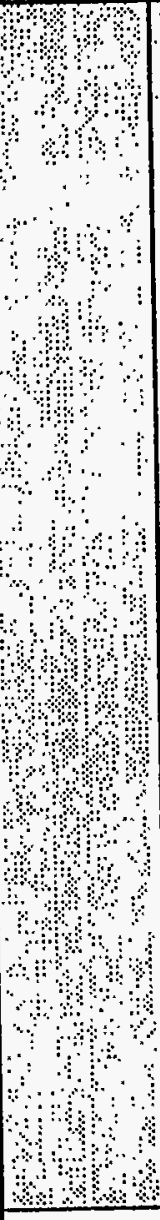 & 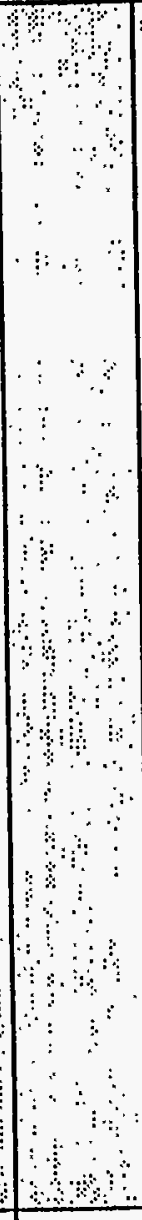 & 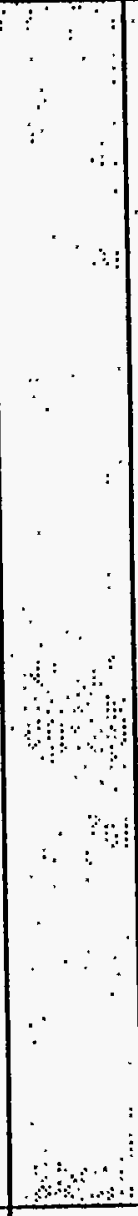 & 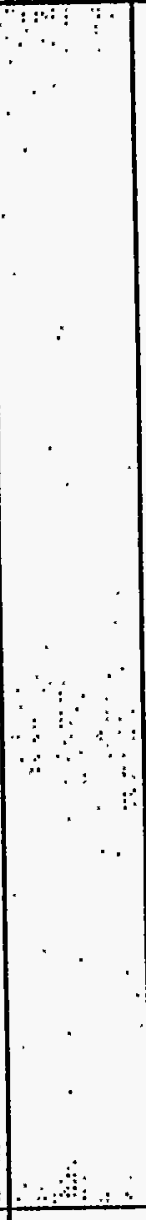 & $\begin{array}{l}\cdot \\
\\
\\
\\
\\
\vdots \\
\vdots \\
\vdots \\
\vdots \\
\vdots \\
\vdots \\
\vdots\end{array}$ \\
\hline 1MDL08 Labor & 74 & 3683 & 3 & 326 & 77 & 4009 & 0 & 120.3 & 77 & 4129.3 \\
\hline 1MDL08 Support From Others and Non-Labor & 8 & 1247 & .8 & -8 & 8.8 & 1239 & 0 & 37.2 & 8.8 & 1276.2 \\
\hline 1MDL08 Total Cost Account & 82 & 4930 & 3.8 & 318 & 85.8 & 5248 & 0 & 157.5 & 85.8 & 5405.5 \\
\hline
\end{tabular}




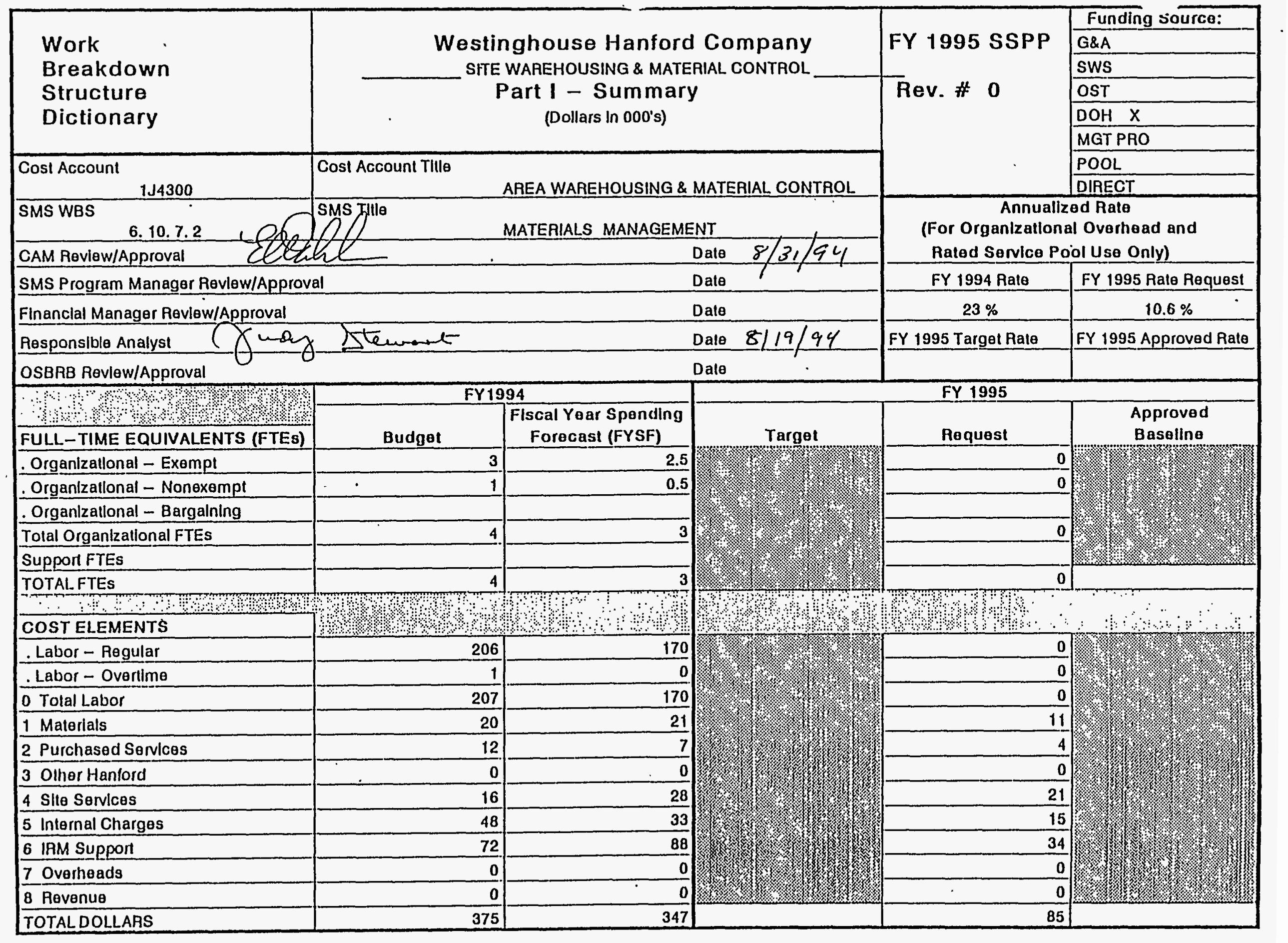




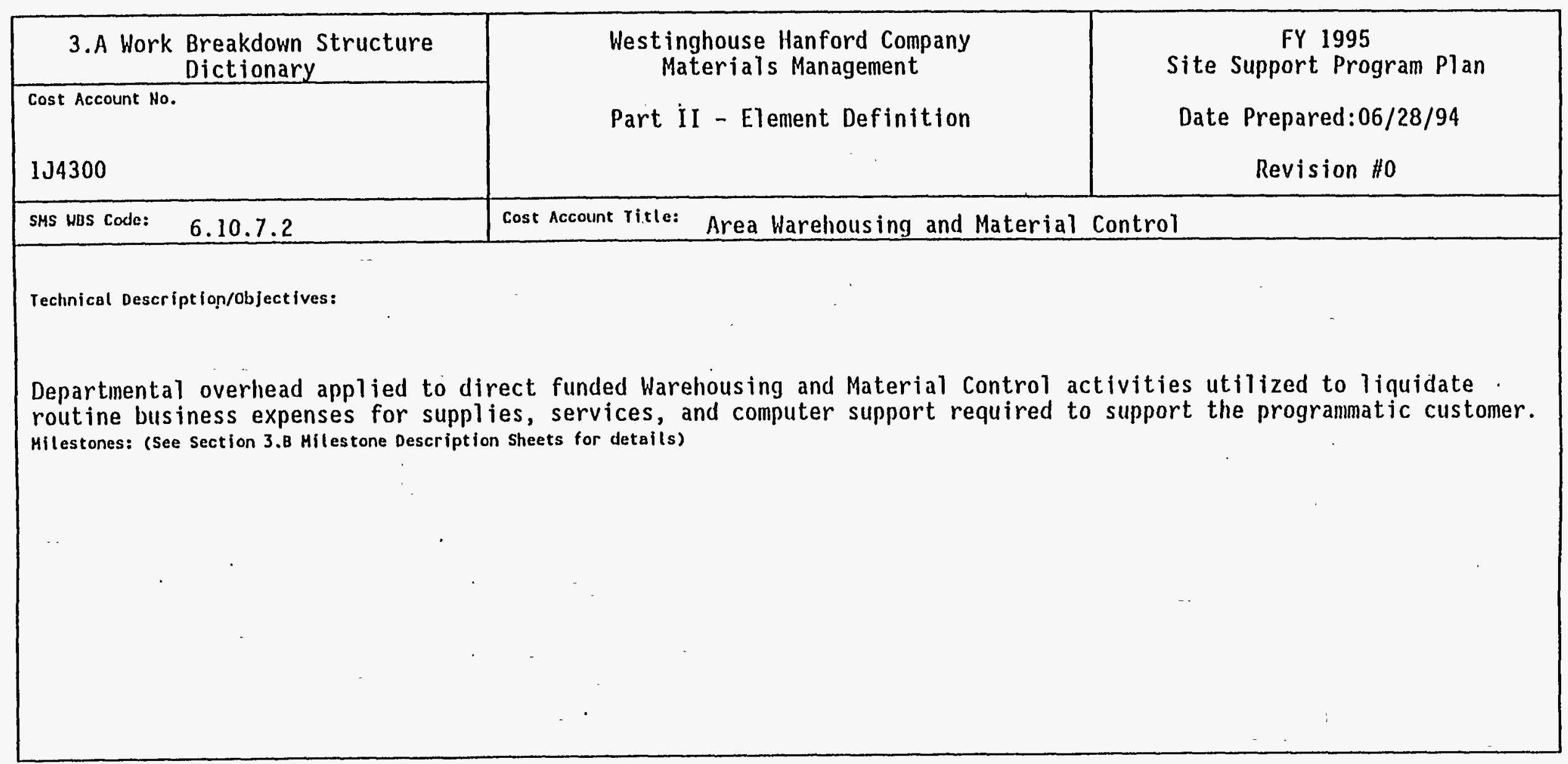




\begin{tabular}{|c|c|c|c|c|c|c|c|c|c|c|}
\hline Cost Account Ho. $1 \mathrm{~J} 4300$ & \multicolumn{6}{|c|}{ Part II - Materials Management } & \multicolumn{4}{|c|}{6.10 .7 .2} \\
\hline Element Task Description & \multicolumn{2}{|c|}{1994 FYSF } & \multicolumn{2}{|c|}{ Delta } & \multicolumn{2}{|c|}{ FY 1995} & \multicolumn{2}{|c|}{ Delta } & \multicolumn{2}{|c|}{ FY 1996} \\
\hline Requests Within Target: & FTEs & $\$ s$ & FTEs & $\$ s$ & FTEs & $\$ s$ & FTES & $\$ s$ & FTES & $\$ s$ \\
\hline $\begin{array}{l}\text { Activity Detalled Description: } \\
\text { 6.10.7.2 - Area Warehousing and Material } \\
\text { Control. }\end{array}$ & 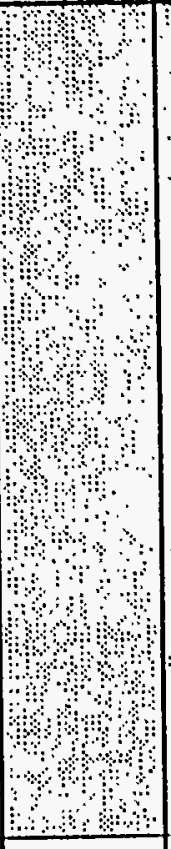 & 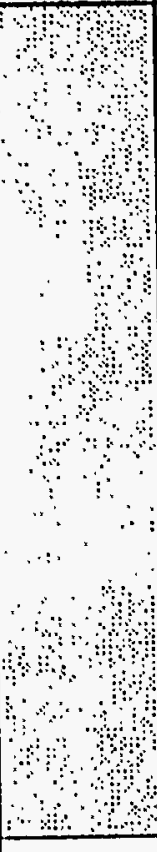 & 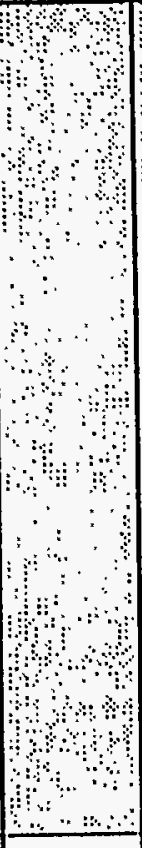 & 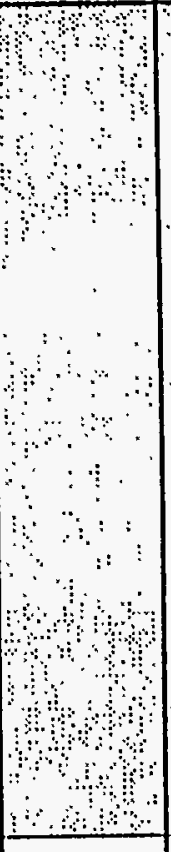 & 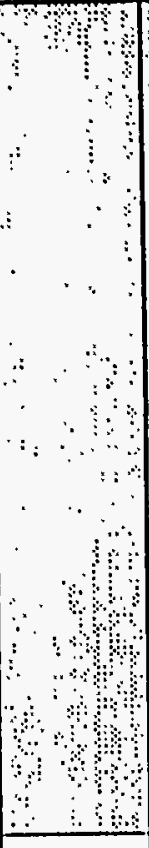 & 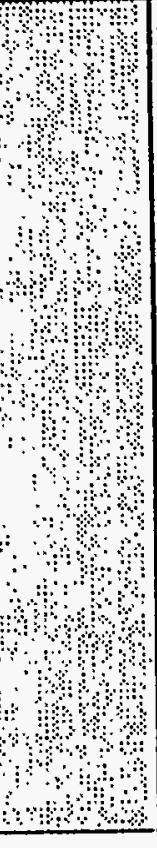 & 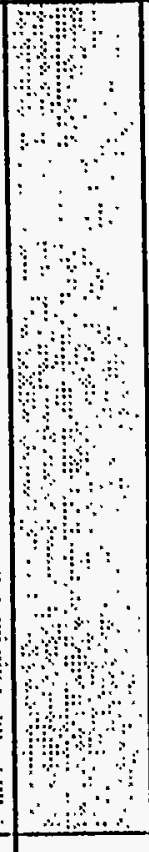 & 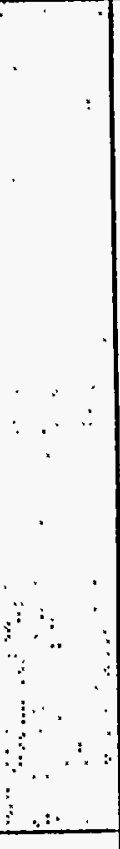 & $\begin{array}{l} \\
\ddots \\
\because \\
\because \\
\vdots \\
\ddots \\
\vdots \\
\because \\
\because\end{array}$ & 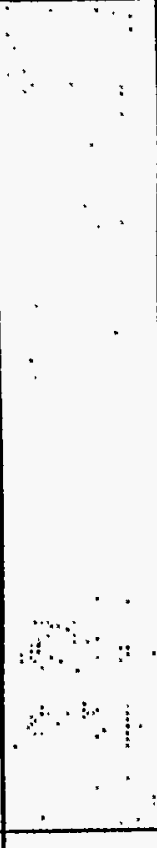 \\
\hline $1 \mathrm{~J} 4300$ Labor & 4 & 207 & -4 & -207 & 0 & 0 & 0 & 0 & 0 & 0 \\
\hline 1J4300 Non-Labor & 0 & 168 & 0 & -83 & 0 & 85 & 0 & 2.6 & 0 & 87.6 \\
\hline $1 \mathrm{~J} 4300$ Total Cost Account & 4 & 375 & -4 & -290 & 0 & 85 & 0 & 2.6 & 0 & 87.6 \\
\hline
\end{tabular}




\begin{tabular}{|c|c|c|c|c|c|c|c|c|c|c|c|c|c|c|c|c|c|}
\hline $\begin{array}{l}\text { Work } \\
\text { Breakdown } \\
\text { Structure } \\
\text { Dictionary }\end{array}$ & \multicolumn{14}{|c|}{$\begin{array}{c}\text { Westinghouse Hanford Company } \\
\text { (insert responsible organizatlon Ille here) } \\
\text { Part III - Liquidation Base Analysis } \\
\text { OR ORGANIZATIONAL OVERHEAD ONLY }\end{array}$} & \multicolumn{3}{|c|}{$\begin{array}{l}\text { FY } 1995 \text { SSPP } \\
\text { Revision \# } 0\end{array}$} \\
\hline $1 J 4300$ & \multicolumn{13}{|c|}{ OUTER AREA WAAEHOUSING \& MATERIAL CONTROL } & & & & \\
\hline DIRECT STAFF* & 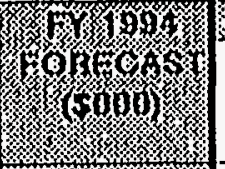 & OCT & NOV & DEC & JAN & FEB & rys & APR & MAY & JUN & JULL & AUG & SEP & $\begin{array}{l}\text { AVG. } \\
\text { DIRECT } \\
\text { STAFF }\end{array}$ & $\begin{array}{c}\text { AVG. } \\
\text { LABOA } \\
\text { AATE } \\
\end{array}$ & $\begin{array}{c}\text { REALIZATION } \\
\text { HOURS }\end{array}$ & $\begin{array}{l}\text { DIRECT } \\
\text { LABOR } \\
(\$ 000)\end{array}$ \\
\hline EXEMPT & & 4 & 4 & 4 & 4 & 4 & 4 & 4 & 4 & 4 & 4 & 4 & 4 & 4 & 32.37 & 1812 & 205 \\
\hline NONEX/PT/TEMP & & 3 & 3 & 3 & 3 & 3 & 3 & 3 & 3 & 3 & 3 & 3 & 3 & 3 & 16.26 & 1812 & 88 \\
\hline BARGAINING & & 12 & 12 & 12 & 12 & 12 & 12 & 12 & 12 & 12 & 12 & 12 & 12 & 12 & $24.37 \ldots$ & 1812 & 508 \\
\hline OVERTIME FACTOR & & & & & 32 & & & & $18=0$ & & & & & & & & -0 \\
\hline DIRECT LABOA BASE & & 18 & 18 & 18 & 18 & 18) & 18 & 18] & 18 & 18 & $18]$ & $18]$ & 18 & 18 & & 2 & 802 \\
\hline ORG OVHD COST & 347 & & & & & & & & 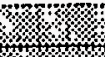 & & & & & & & & $-8 \underline{5}$ \\
\hline DIAECT LABOR BASE & 1,538 & & & & & & & & & & & & & & & & 802 \\
\hline RATE (COST/BASE) & $22.5 \%$ & 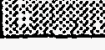 & & & & & & & & & & & & & & & $10.6 \%$ \\
\hline
\end{tabular}




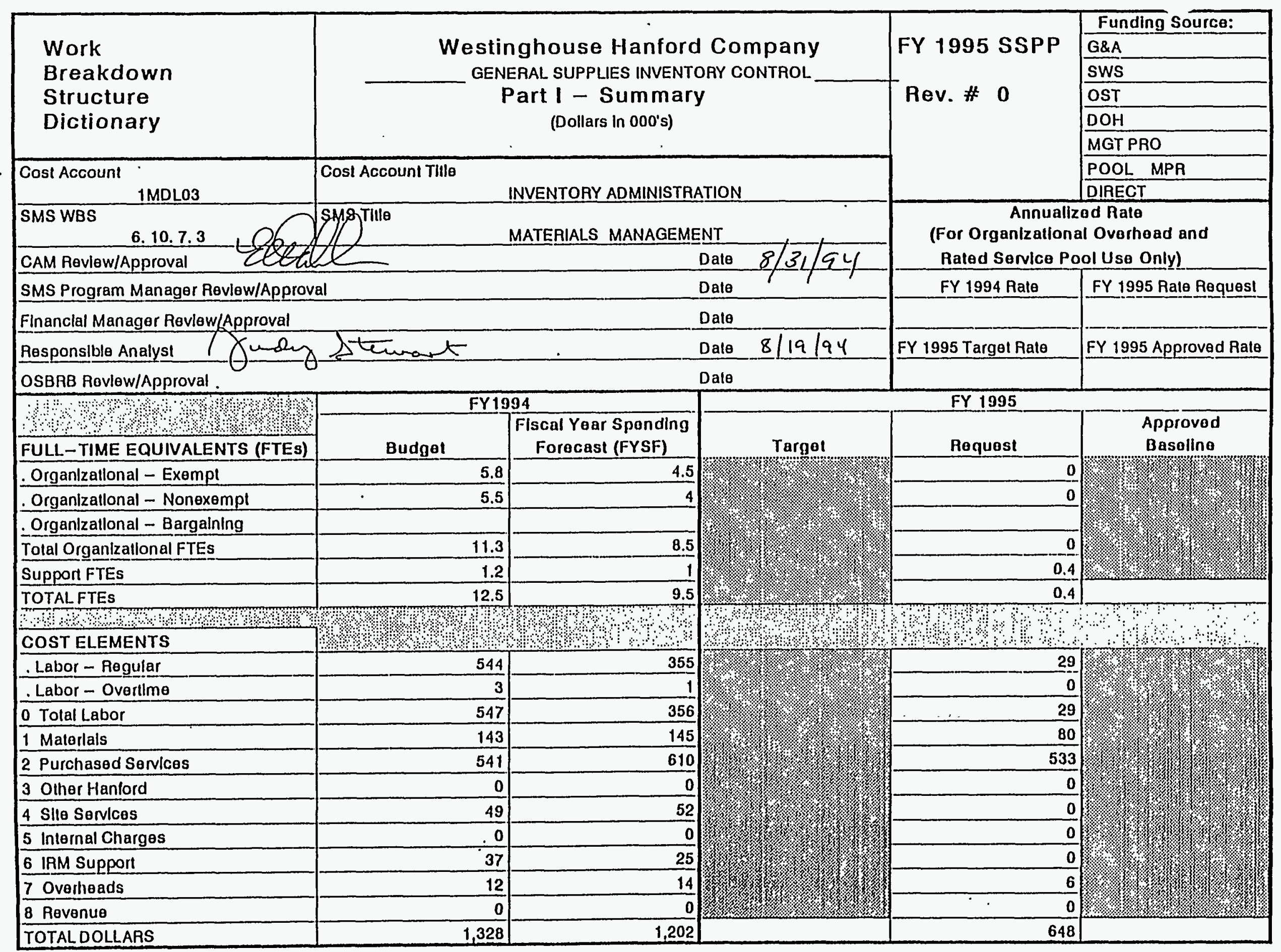




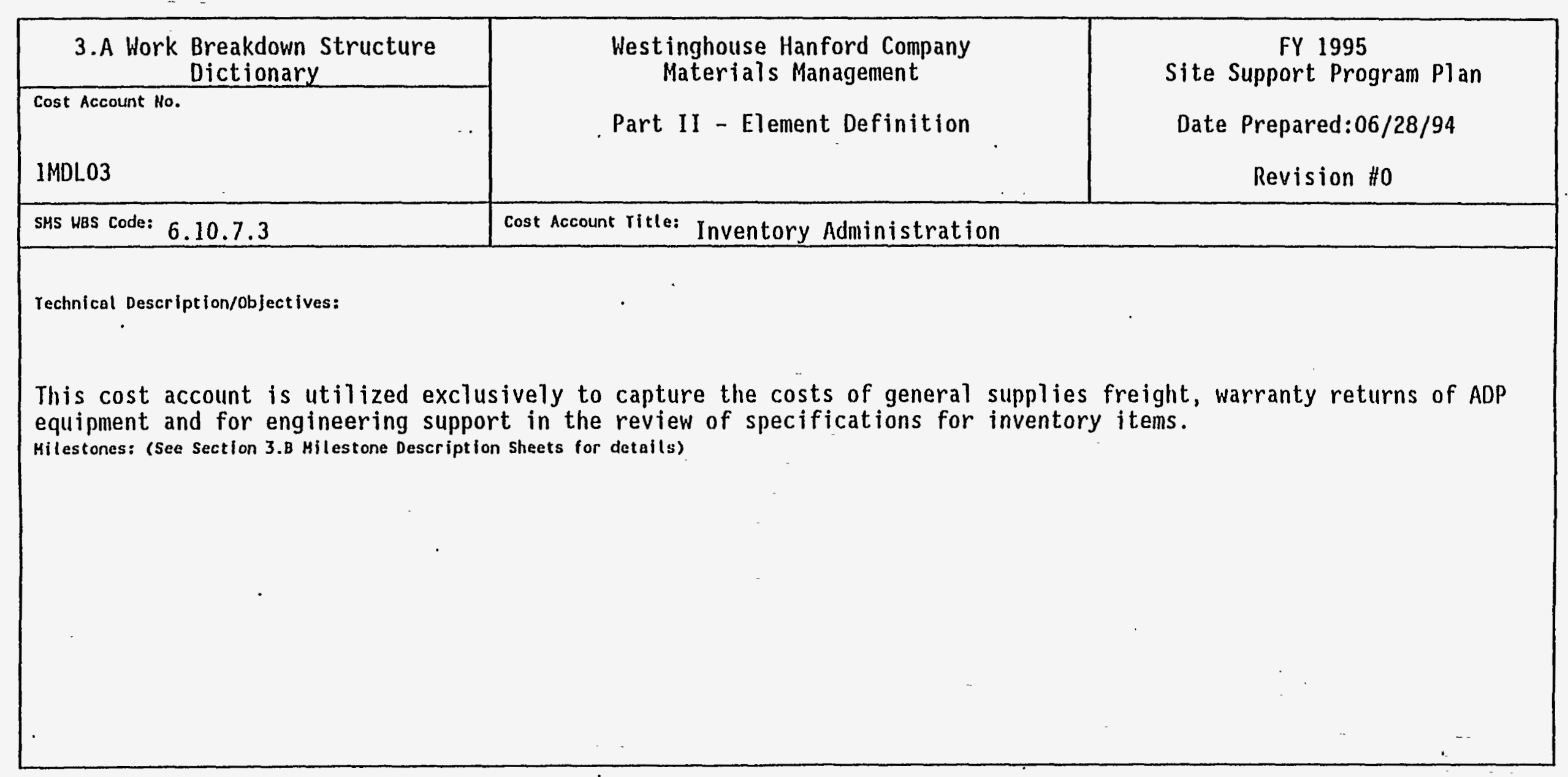




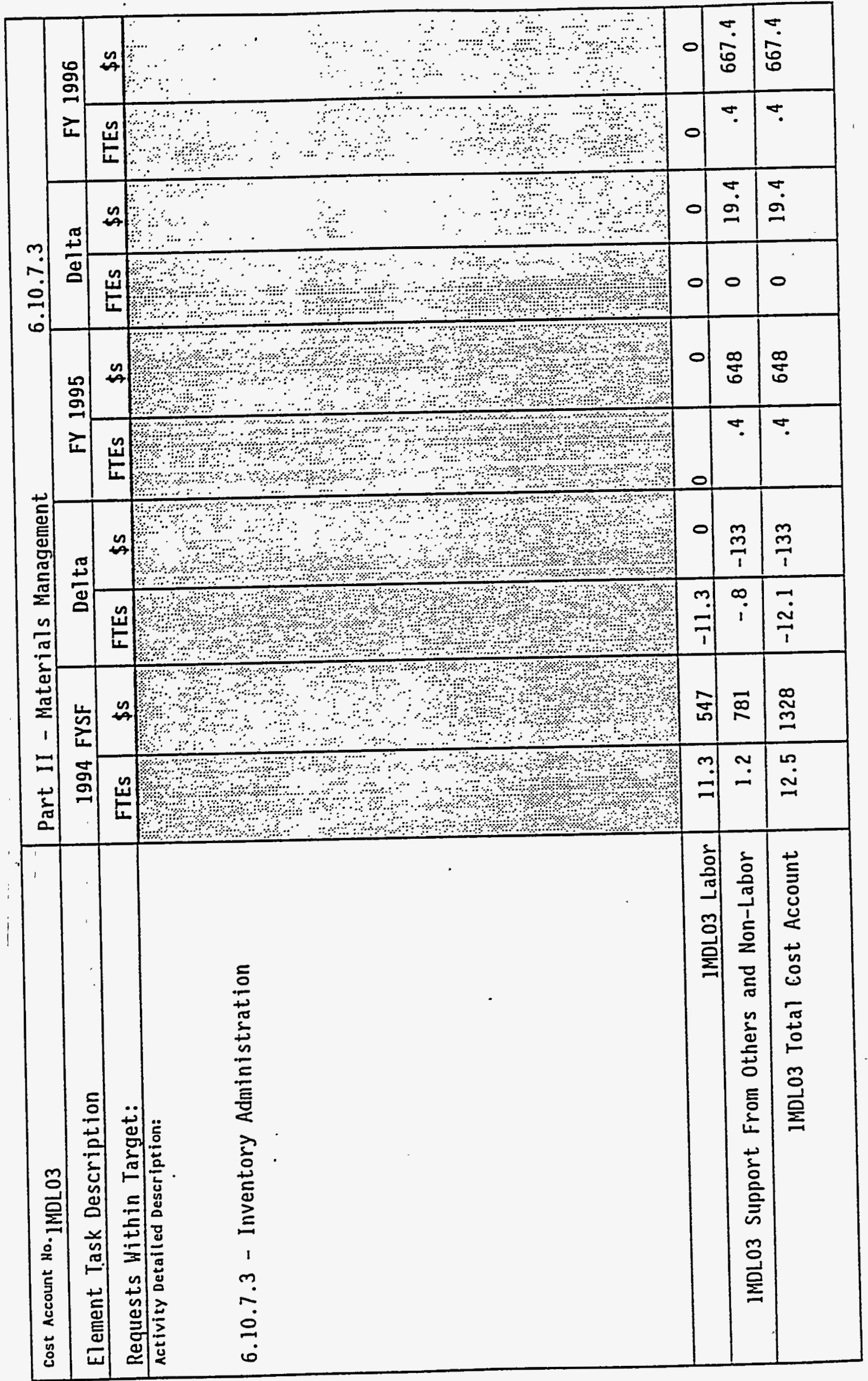

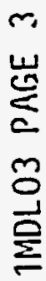




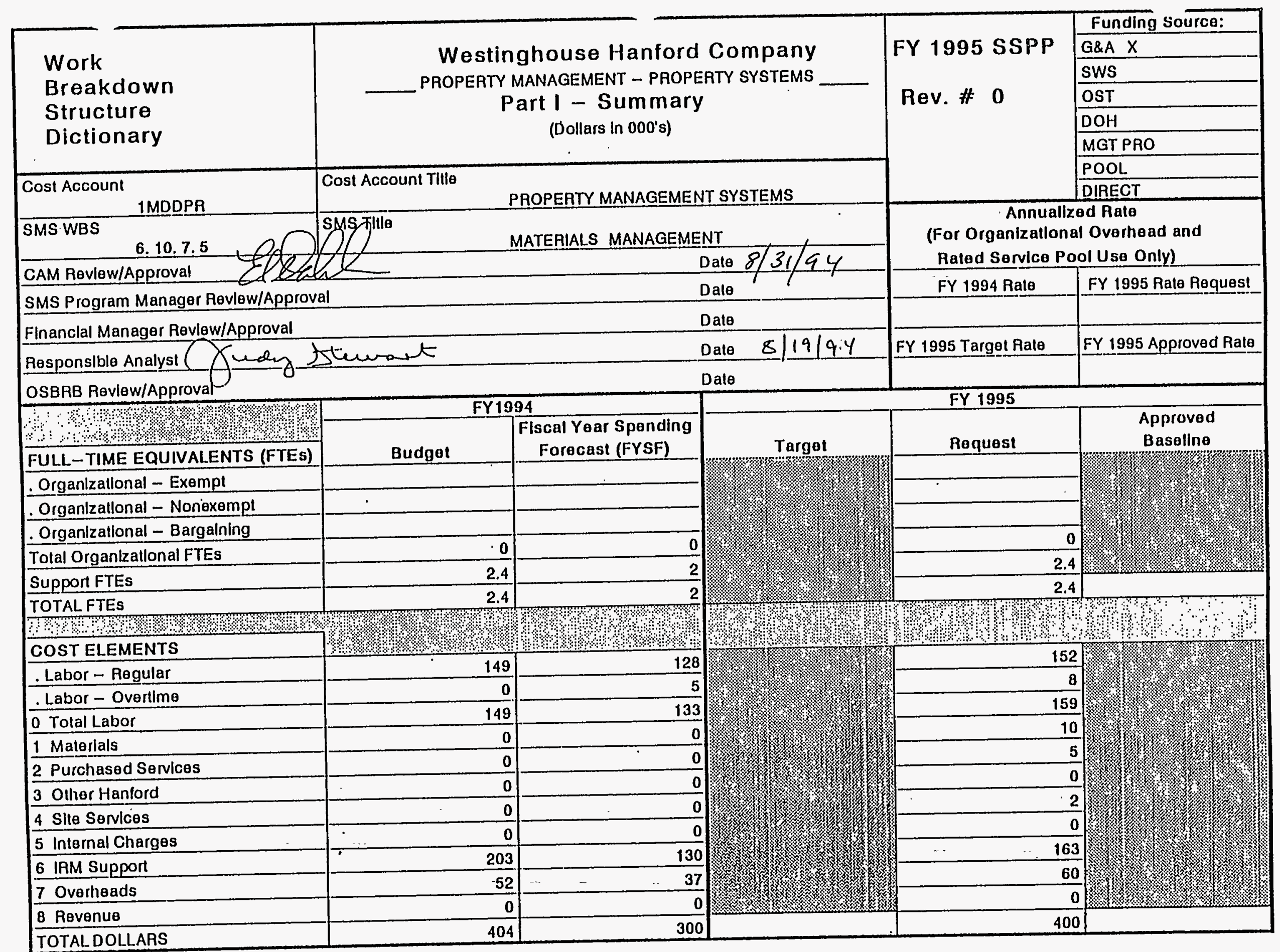




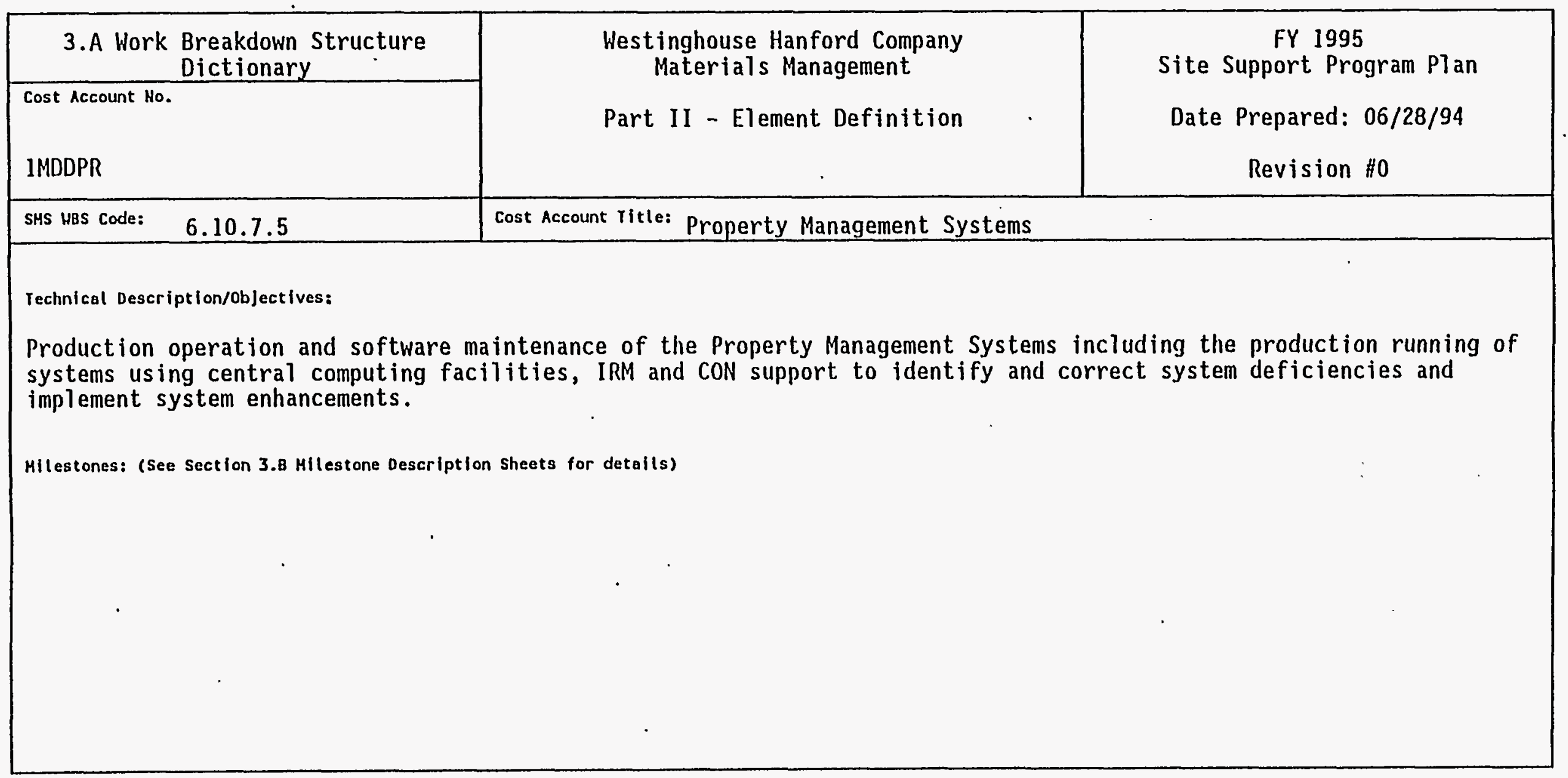




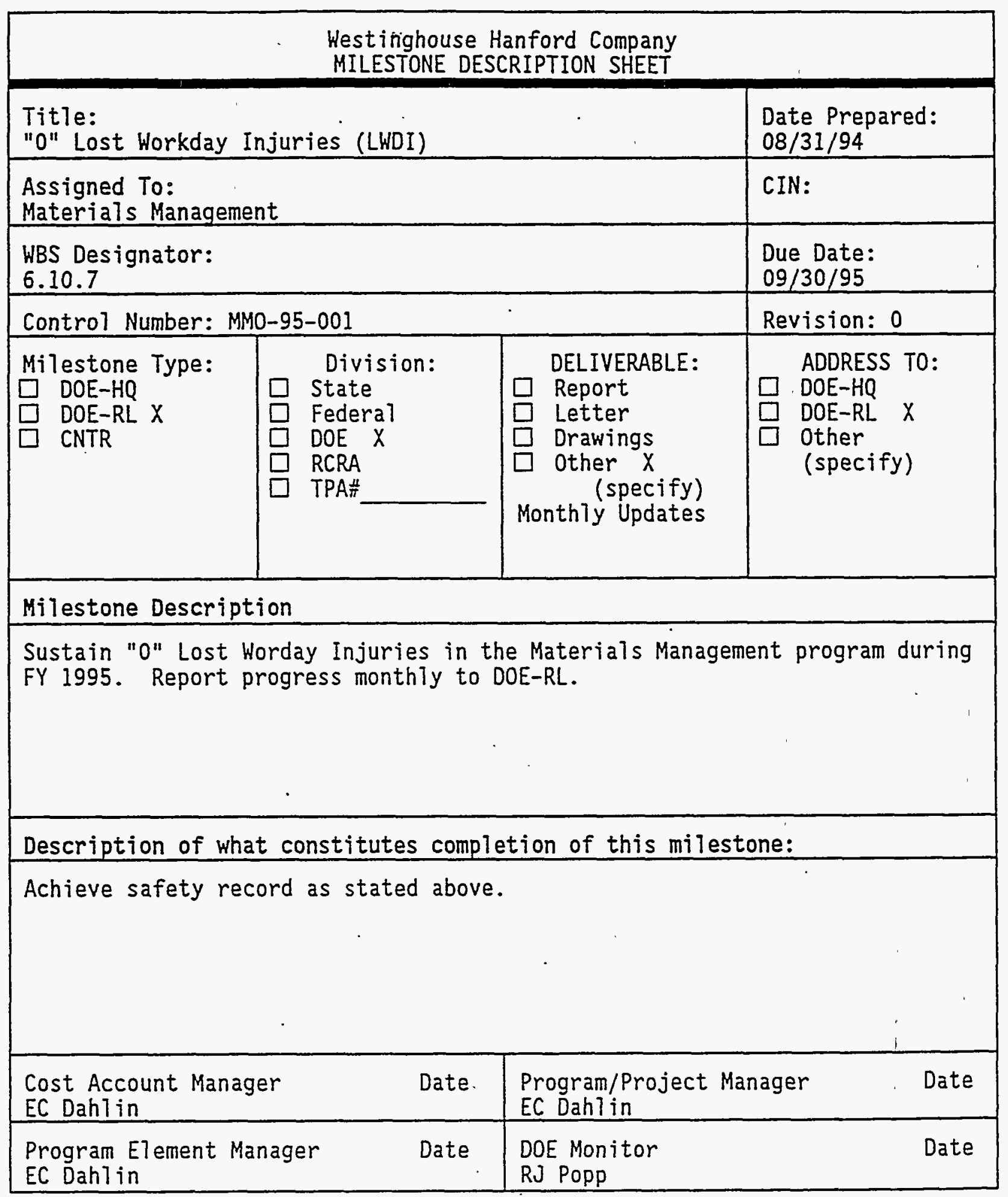




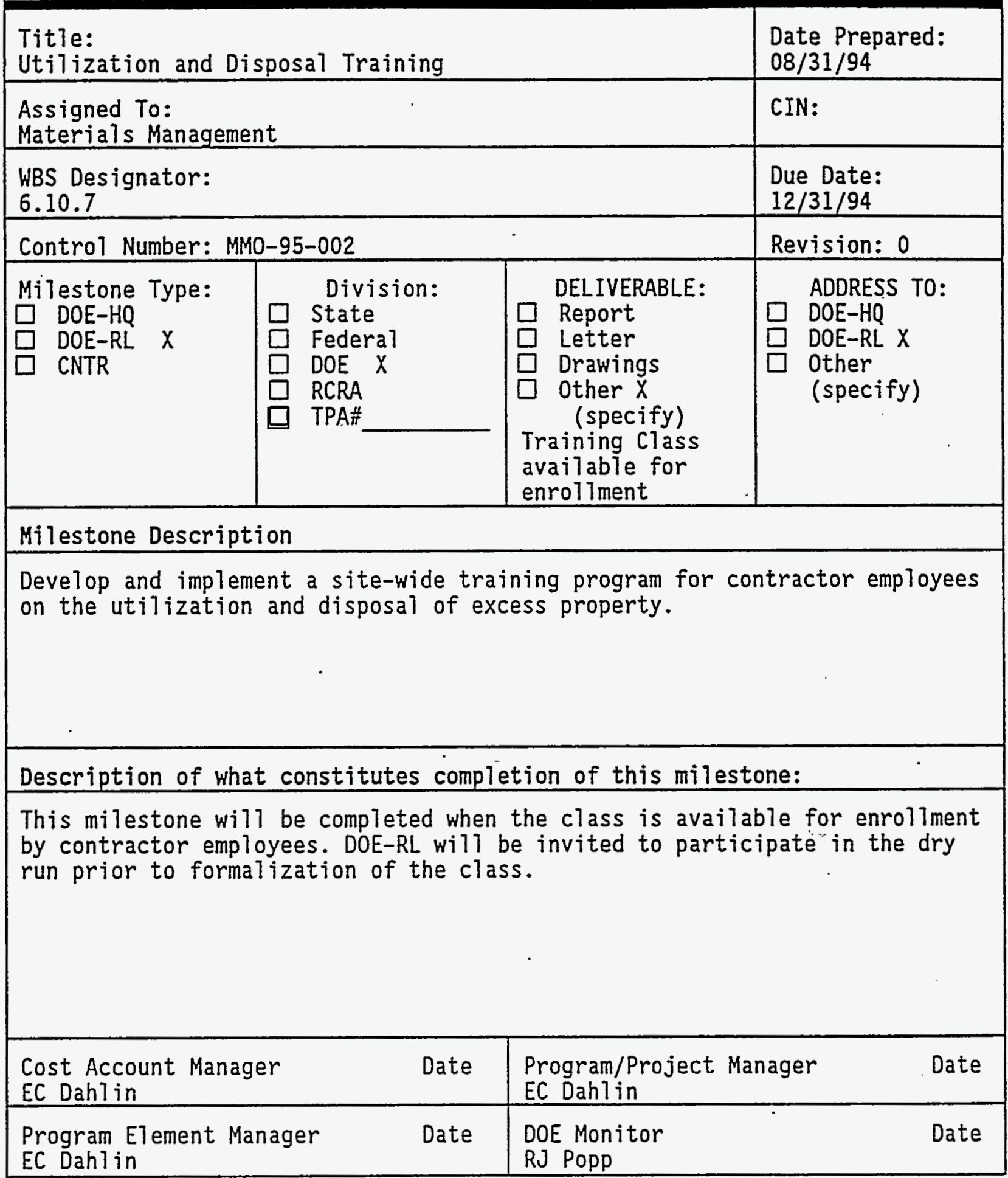




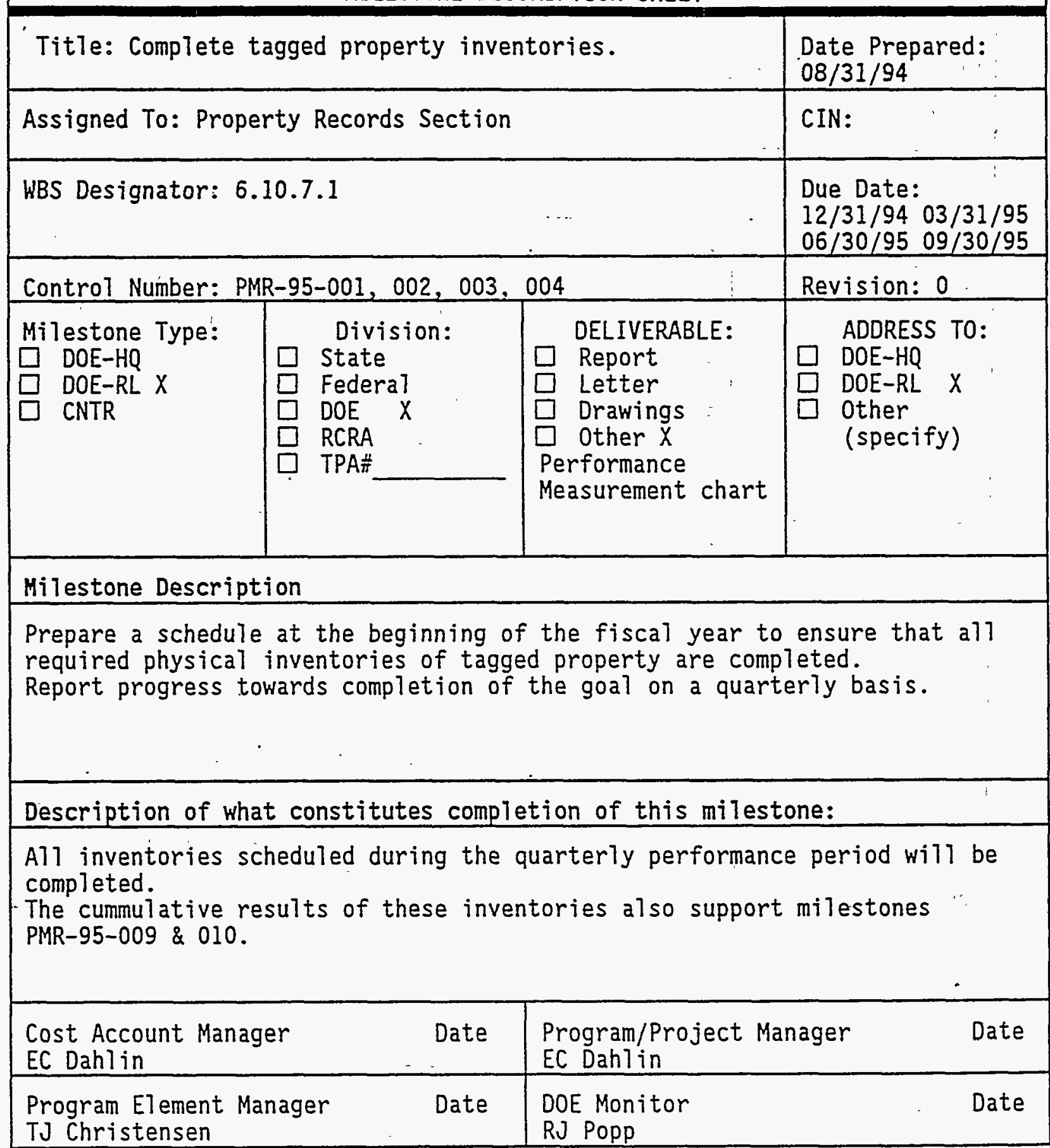




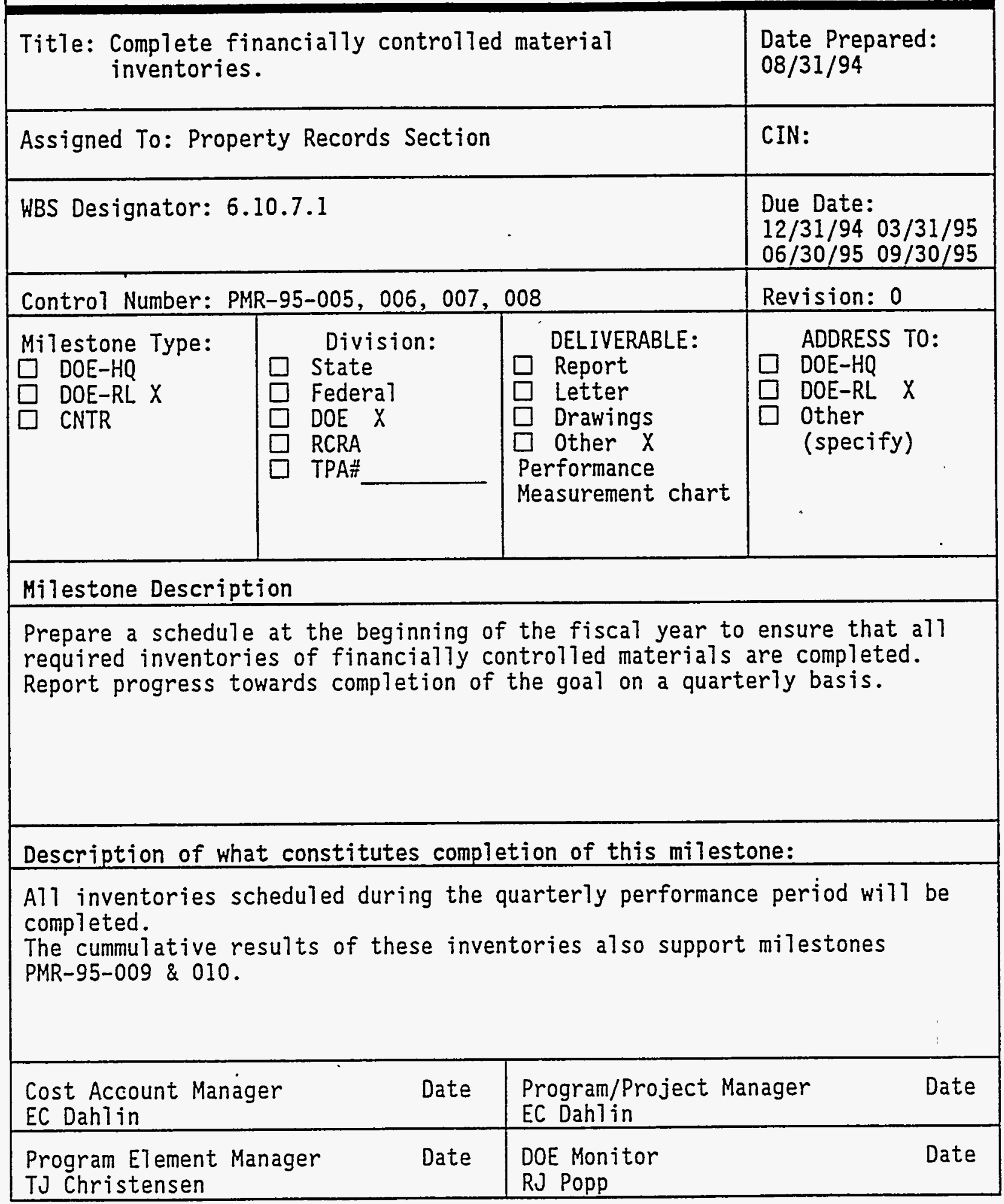




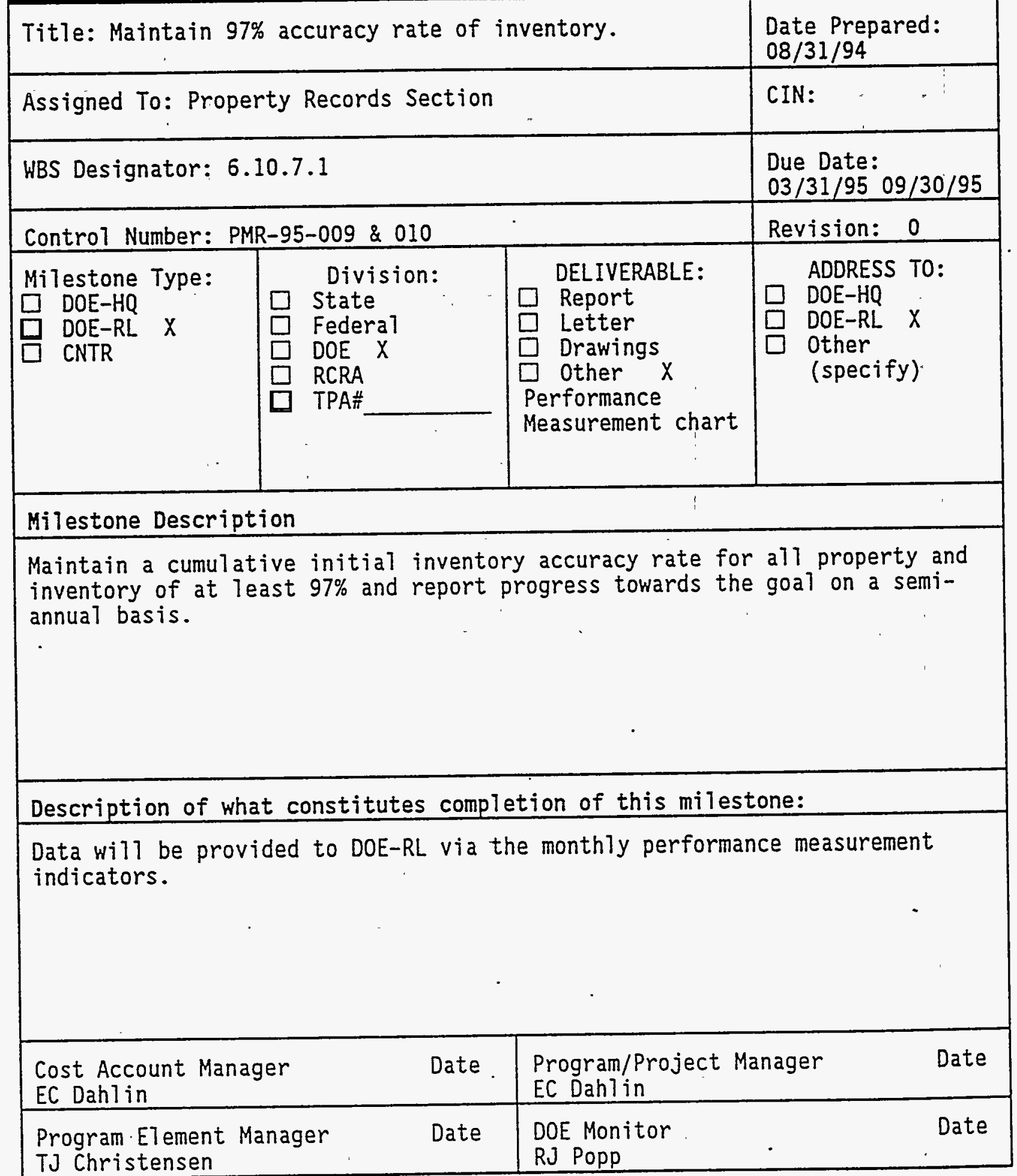




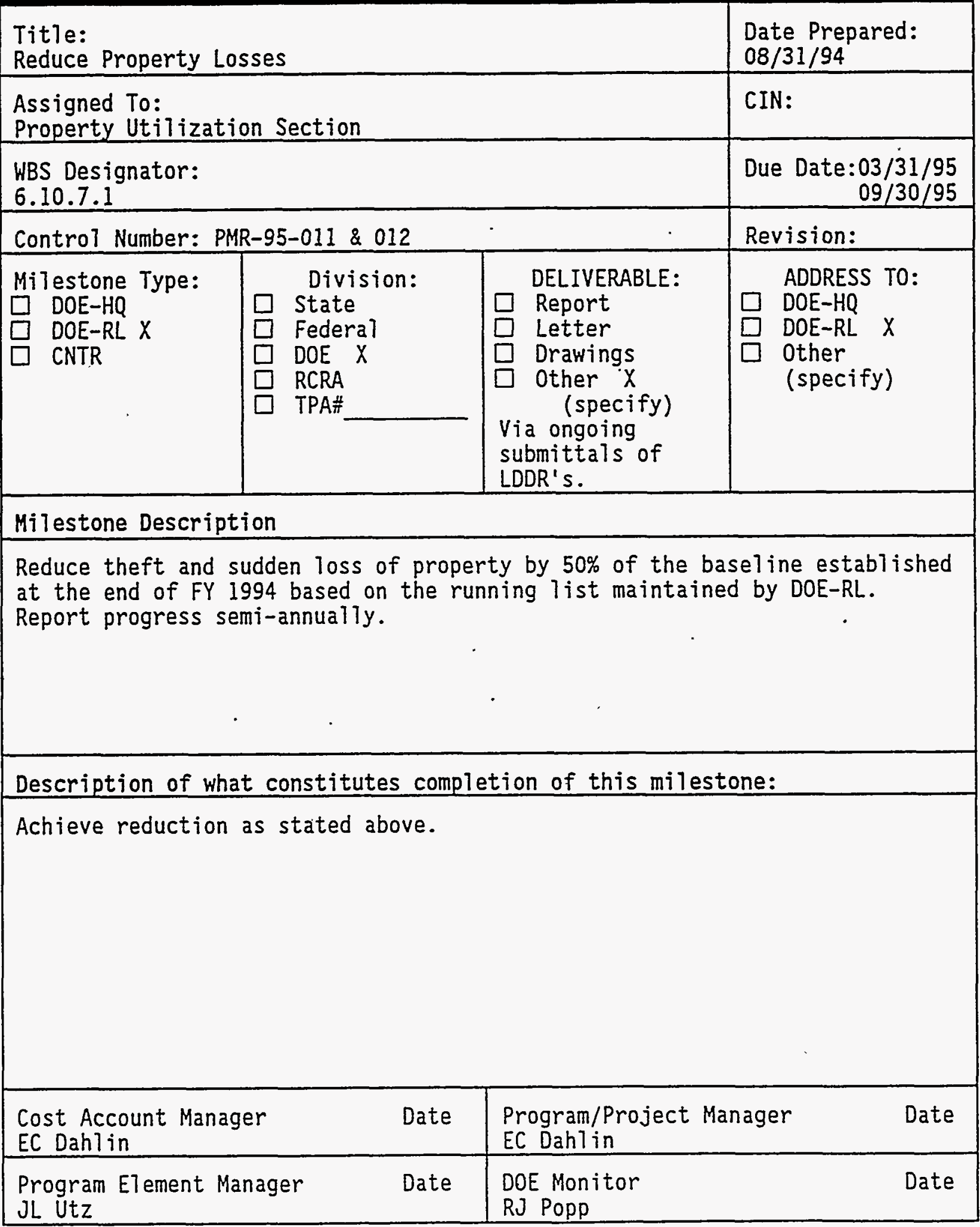


Westinghouse Hanford Company MILESTONE DESCRIPTION SHEET

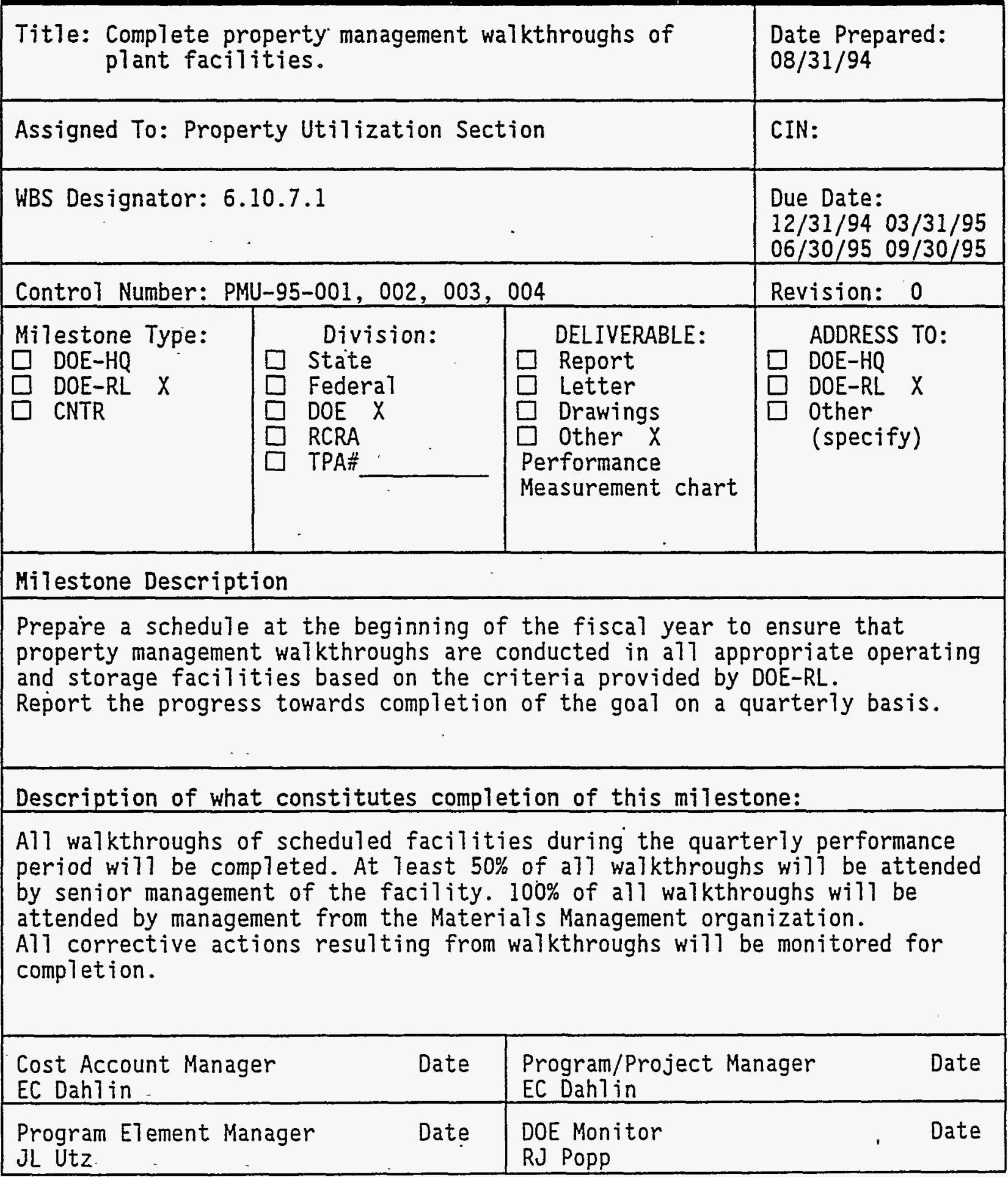




\begin{tabular}{|c|c|c|c|c|c|}
\hline \multicolumn{6}{|c|}{$\begin{array}{l}\text { Westinghouse Hanford Company } \\
\text { MILESTONE DESCRIPTION SHEET }\end{array}$} \\
\hline \multicolumn{4}{|c|}{$\begin{array}{l}\text { Title: } \\
\$ 3 M \text { in Utilization of offsite Excess }\end{array}$} & $\begin{array}{l}\text { Date } \\
08 / 3\end{array}$ & \\
\hline \multicolumn{4}{|c|}{$\begin{array}{l}\text { Assigned To: } \\
\text { Property Utilization Section }\end{array}$} & CIN: & \\
\hline \multicolumn{4}{|l|}{$\begin{array}{l}\text { WBS Designator: } \\
6.10 .7 .1\end{array}$} & $\begin{array}{l}\text { Due } \\
09 / 3\end{array}$ & \\
\hline \multicolumn{4}{|c|}{ Control Number: PMU-95-005 } & Revi & \\
\hline $\begin{array}{l}\text { MiTestone Type: } \\
\square \text { DOE-HQ } \\
\square \text { DOE-RL X } \\
\square \text { CNTR }\end{array}$ & $\begin{array}{ll}\square & S \\
\square & f \\
\square & D \\
\square & R \\
\square & T\end{array}$ & & $\begin{array}{l}\text { DELIVERABLE: } \\
\square \text { Report } \\
\square \text { Letter } \\
\square \text { Drawings } \\
\square \text { Other X } \\
\text { (specify) } \\
\text { Monthly } \\
\text { performance } \\
\text { indicator }\end{array}$ & $\begin{array}{ll}A \\
\square \\
\square \\
\square \\
\square \\
0 \\
1\end{array}$ & \\
\hline \multicolumn{6}{|c|}{ Milestone Description } \\
\hline \multicolumn{6}{|c|}{$\begin{array}{l}\text { Acquire } \$ 3 M \text { in excess property through offsite acquisition from other DOE } \\
\text { Sites and Federal Agencies. Report progress via the monthly Performance } \\
\text { Measurement Indicators. }\end{array}$} \\
\hline \multicolumn{6}{|c|}{ Description of what constitutes completion of this milestone: } \\
\hline \multicolumn{6}{|c|}{ Achieve reutilization totals stated above by fiscal year end 1995 . } \\
\hline \multicolumn{2}{|c|}{$\begin{array}{l}\text { Cost Account Manager } \\
\text { EC Dahlin }\end{array}$} & Date & $\begin{array}{l}\text { Program/Project } \\
\text { EC Dahlin }\end{array}$ & nager & Date \\
\hline \multicolumn{2}{|c|}{$\begin{array}{l}\text { Program Element Manager } \\
\text { JL Utz }\end{array}$} & Date & $\begin{array}{l}\text { DOE Monitor } \\
\text { RJ Popp }\end{array}$ & & Date \\
\hline
\end{tabular}




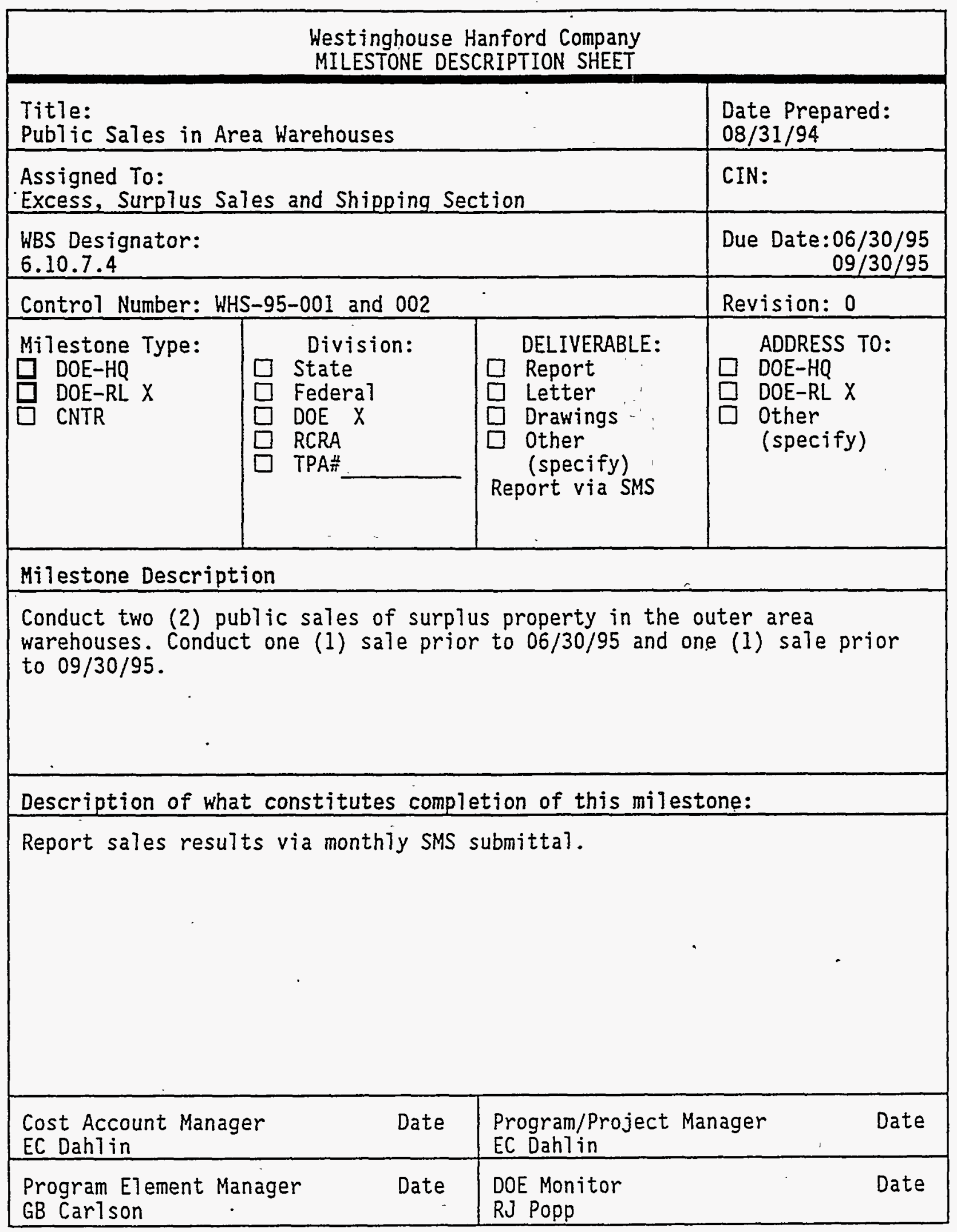




\begin{tabular}{|c|c|c|c|}
\hline \multicolumn{4}{|c|}{$\begin{array}{l}\text { Westinghouse Hanford Company } \\
\text { MILESTONE DESCRIPTION SHEET }\end{array}$} \\
\hline \multicolumn{3}{|c|}{$\begin{array}{l}\text { Title: } \\
\text { Systems Contracting Activity }\end{array}$} & $\begin{array}{l}\text { Date Prepared: } \\
08 / 31 / 95\end{array}$ \\
\hline \multicolumn{3}{|c|}{$\begin{array}{l}\text { Assigned To: } \\
\text { Systems Contract Management Team }\end{array}$} & CIN: \\
\hline \multicolumn{3}{|c|}{$\begin{array}{l}\text { WBS Designator: } \\
6.10 .7 .3\end{array}$} & $\begin{array}{r}\text { Due Date: } 12 / 31 / 94 \\
03 / 31 / 95 \\
06 / 30 / 95 \\
09 / 30 / 95 \\
\end{array}$ \\
\hline \multicolumn{3}{|c|}{ Control Number: IMS-95-001, 002, 003, \& 004} & Revision:0 \\
\hline $\begin{array}{l}\text { Milestone Type: } \\
\square \text { DOE-HQ } \\
\square \text { DOE-RL X } \\
\square \text { CNTR }\end{array}$ & $\begin{array}{ll} & \text { Division: } \\
\square & \text { State } \\
& \text { Federal } \\
\square & \text { DOE X } \\
\square & \text { RCRA } \\
\square & \text { TPA\# } \\
\end{array}$ & $\begin{array}{c}\text { DELIVERABLE: } \\
\square \text { Report } \\
\square \text { Letter } \\
\square \text { Drawings } \\
\square \text { Other X } \\
\quad \text { (specify) } \\
\text { Via monthly SMS }\end{array}$ & $\begin{array}{l}\text { ADDRESS TO: } \\
\square \text { DOE-HQ } \\
\square \text { DOE-RL' } x \\
\square \text { Other } \\
\text { (specify) }\end{array}$ \\
\hline \multicolumn{4}{|c|}{ Milestone Description } \\
\hline \multicolumn{4}{|c|}{$\begin{array}{l}\text { Develop and maintain a quarterly schedule of Systems Contracting and } \\
\text { progress towards completion. }\end{array}$} \\
\hline \multicolumn{4}{|c|}{ Description of what constitutes completion of this milestone: } \\
\hline \multicolumn{4}{|c|}{$\begin{array}{l}\text { Milestone commitment is considered complete when the activities are } \\
\text { completed in accordance with the schedule and or progress is. accepted by } \\
\text { the Program Manager. }\end{array}$} \\
\hline \multicolumn{2}{|c|}{$\begin{array}{l}\text { Cost Account Manager } \\
\text { EC Dahl in }\end{array}$} & \multicolumn{2}{|c|}{$\begin{array}{l}\text { Program/Project Manager } \quad \text { Date } \\
\text { EC Dahl in }\end{array}$} \\
\hline \multicolumn{2}{|c|}{$\begin{array}{l}\text { Program El ement Manager } \\
\text { JG Caudi1T }\end{array}$} & \multicolumn{2}{|c|}{$\begin{array}{ll}\text { DOE Monitor } & \text { Date } \\
\text { RJ Popp } & \\
\end{array}$} \\
\hline
\end{tabular}




\begin{tabular}{|c|c|c|c|}
\hline \multicolumn{3}{|c|}{$\begin{array}{l}\text { Title: } \\
\text { Spare Parts Annual Reviews }\end{array}$} & $\begin{array}{l}\text { Date Prepared: } \\
08 / 31 / 95\end{array}$ \\
\hline \multicolumn{3}{|c|}{$\begin{array}{l}\text { Assigned To: } \\
\text { Inventory Management }\end{array}$} & CIN: \\
\hline \multicolumn{3}{|l|}{$\begin{array}{l}\text { WBS Designator: } \\
6.10 .7 .3\end{array}$} & $\begin{array}{r}\text { Due Date:03/31/95 } \\
09 / 30 / 95\end{array}$ \\
\hline \multicolumn{3}{|c|}{ Control Number: IMS-95-005 and 006} & Revision: 0 \\
\hline $\begin{array}{l}\text { Milestone Type: } \\
\square \text { DOE-HQ } \\
\square \text { DOE-RL X } \\
\square \text { CNTR }\end{array}$ & $\begin{array}{l}\quad \text { Division: } \\
\square \text { State } \\
\square \text { Federal } \\
\square \text { DOE X } \\
\square \text { RCRA } \\
\square \text { TPA\# }\end{array}$ & $\begin{array}{c}\text { DELIVERABLE: } \\
\square \text { Report } \\
\square \text { Letter } \\
\square \text { Drawings } \\
\square \text { Other } X \\
\text { (specify) } \\
\text { Via monthly SMS }\end{array}$ & $\begin{array}{ll} & \text { ADDRESS TO: } \\
\square & \text { DOE-HQ } \\
\square & \text { DOE-RL X } \\
\square & \text { Other } \\
\text { (specify) }\end{array}$ \\
\hline
\end{tabular}

\section{Milestone Description}

Complete the ongoing processes required to rejustify Spare Parts Inventory for retention in accordance with Property Management Regulations. Report progress semi-annualiy.

Description of what constitutes completion of this milestone:

Milestone completion will be reported via monthly SMS.

Data will be provided to DOE-RL to substantiate the validity of the review process.

\begin{tabular}{|ll|ll|}
\hline $\begin{array}{l}\text { Cost Account Manager } \\
\text { EC Dahlin }\end{array}$ & Date & $\begin{array}{l}\text { Program/Project } \\
\text { EC Dahlin }\end{array}$ & Date \\
\hline $\begin{array}{l}\text { Program Element Manager } \\
\text { RE Boykin }\end{array}$ & Date & $\begin{array}{l}\text { DOE Monitor } \\
\text { RJ Popp }\end{array}$ & Date \\
\hline
\end{tabular}




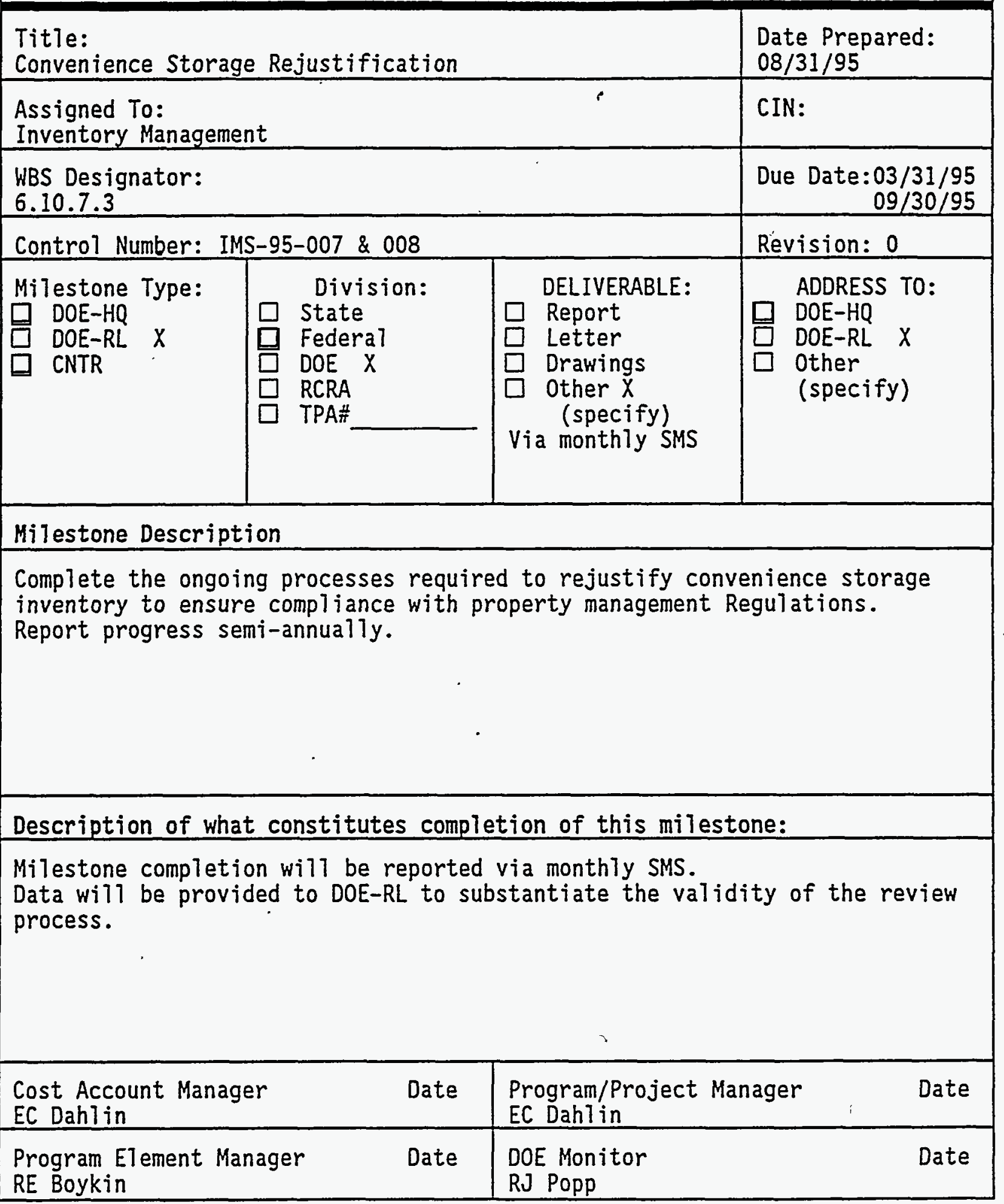




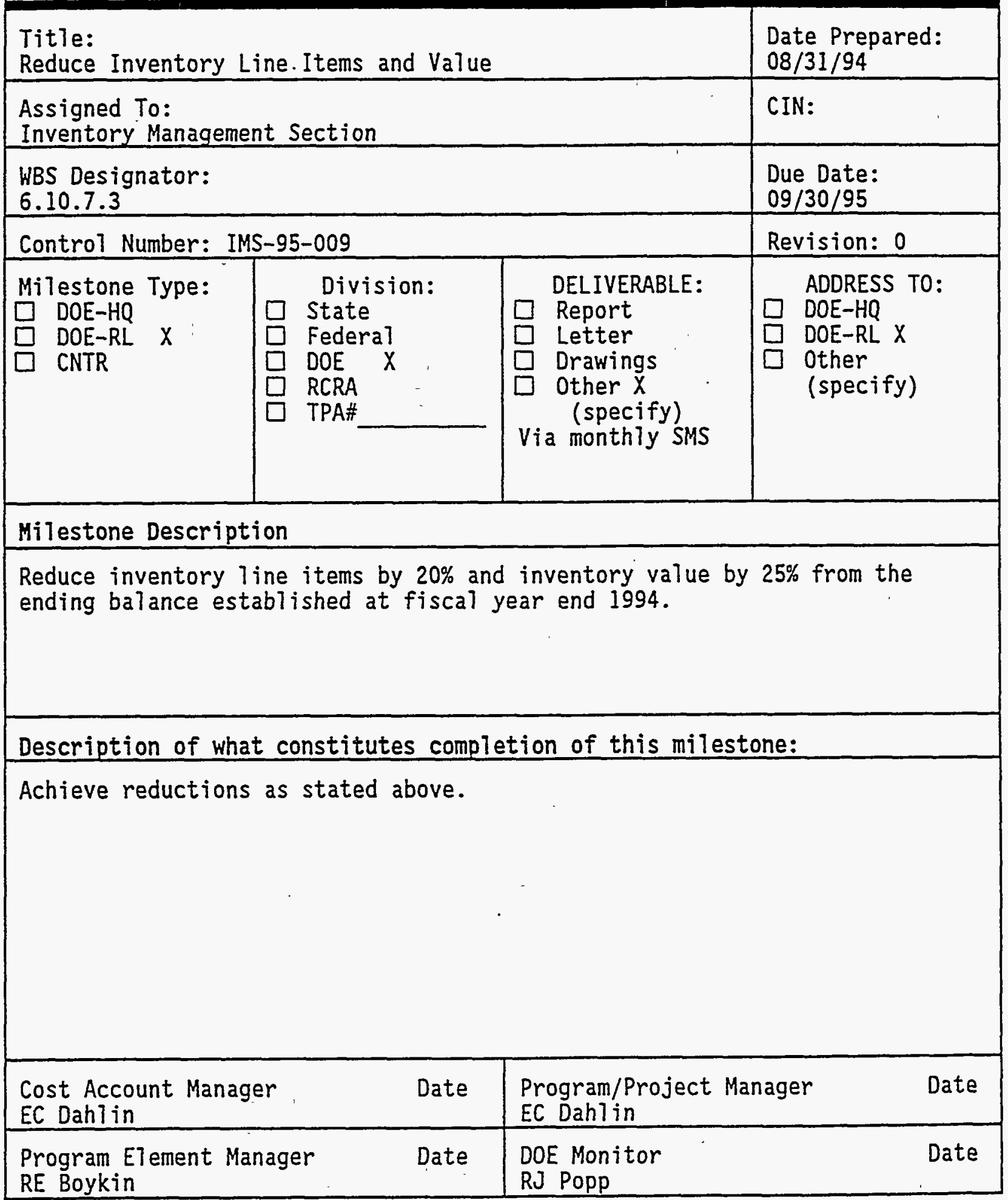




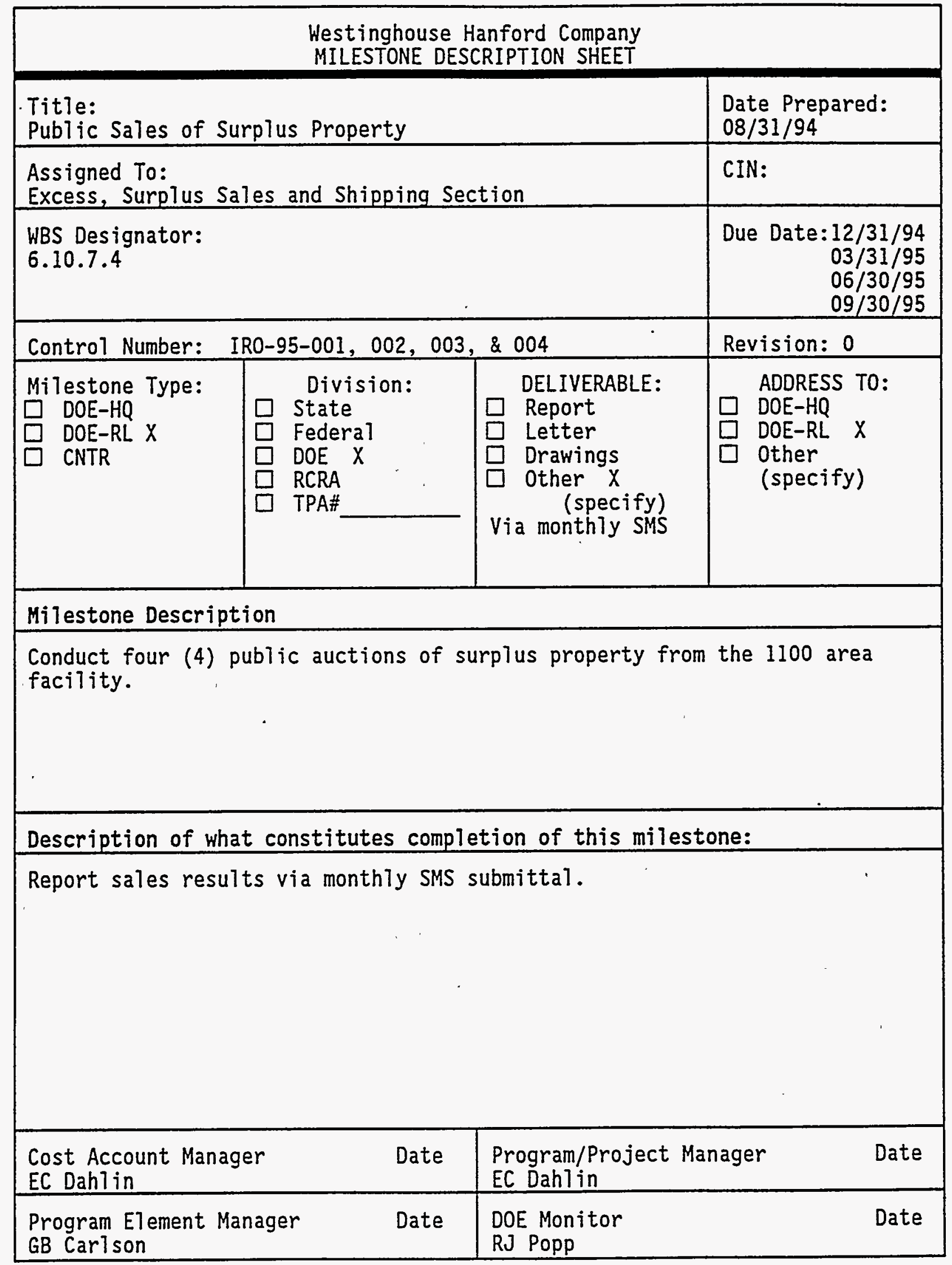


Westinghouse Hanford Company

MILESTONE DESCRIPTION SHEET

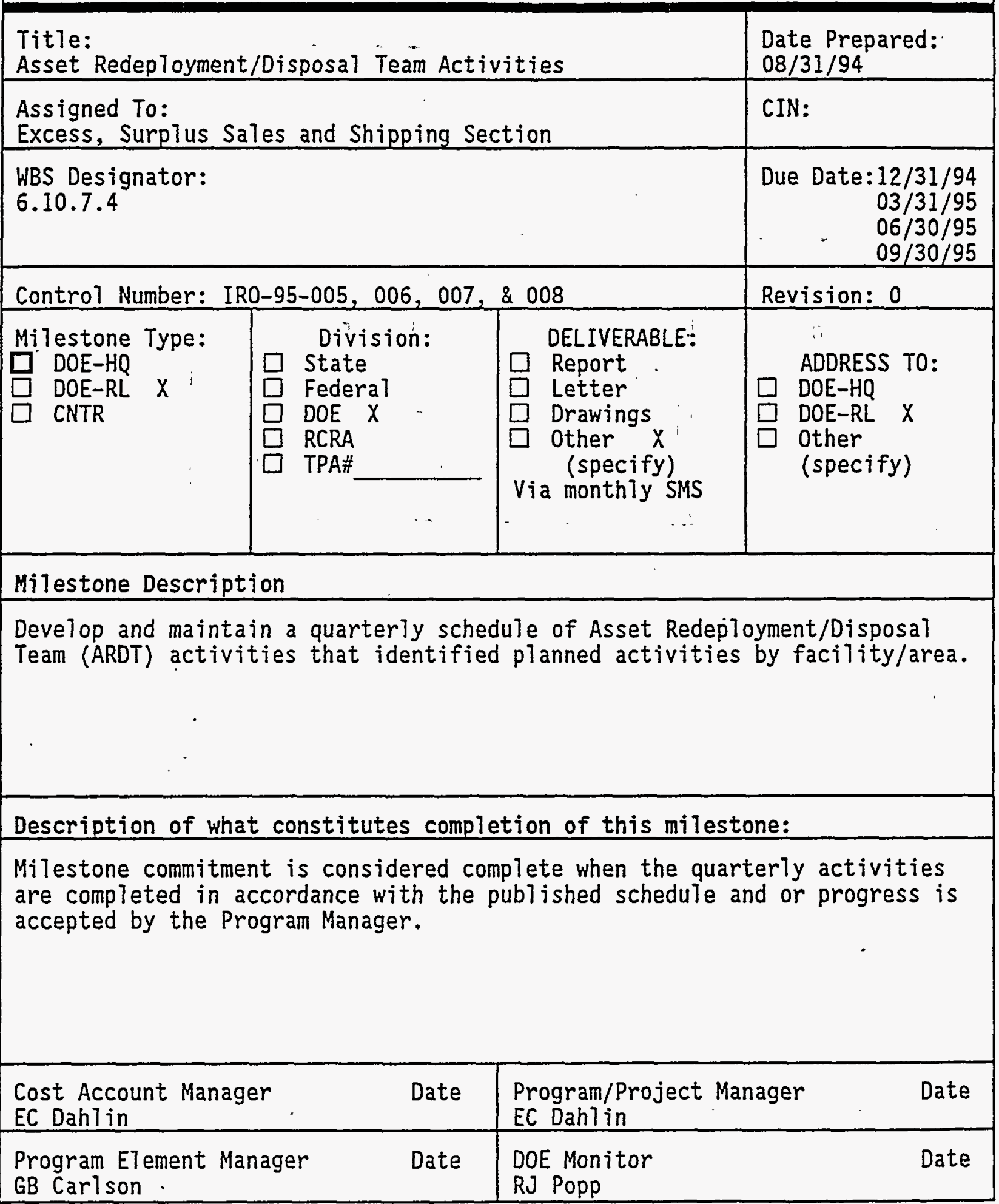




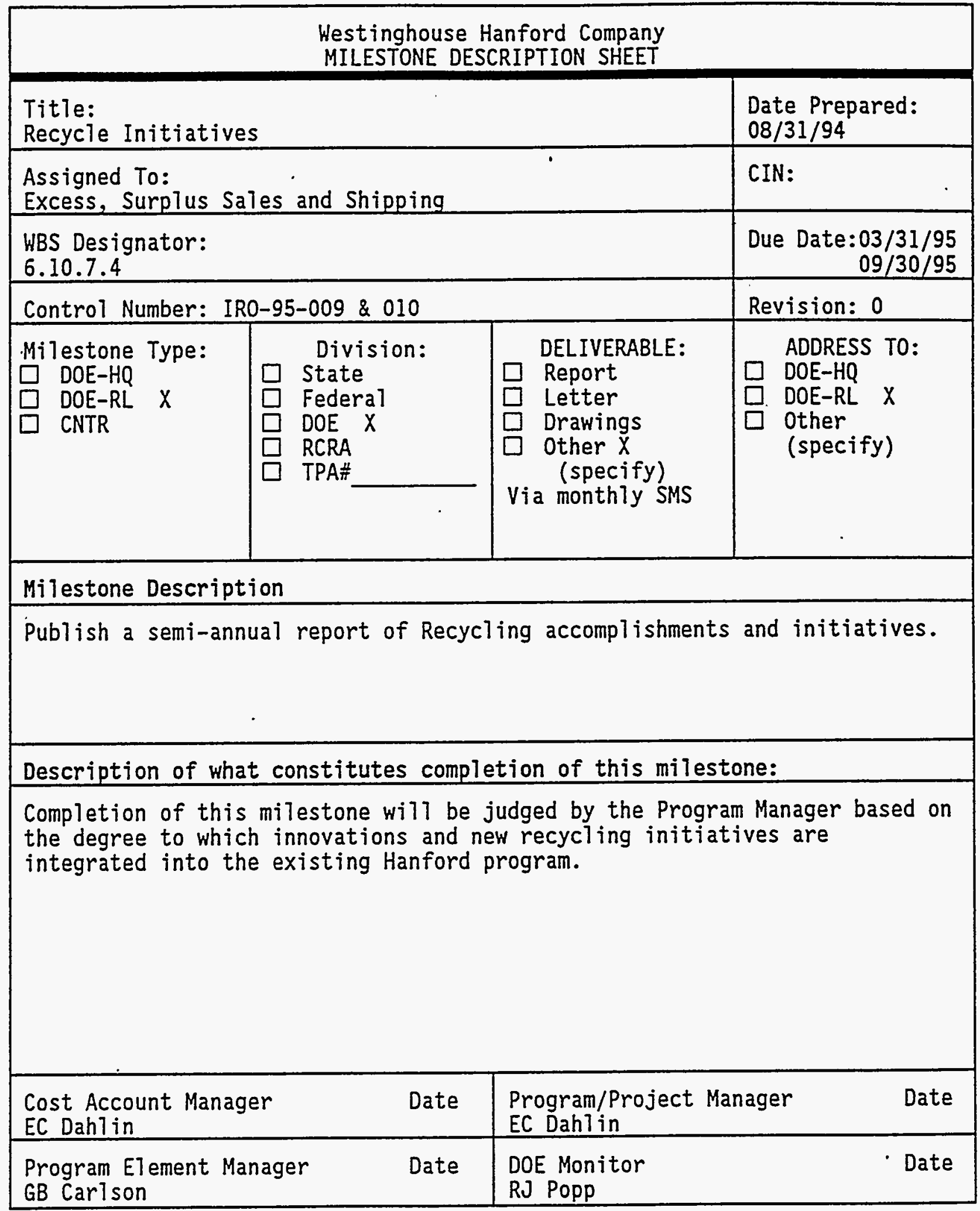


